\title{
CAD model based tracking and 3D visual-based control for MEMS microassembly
}

\author{
B. Tamadazte ${ }^{1}$, E. Marchand ${ }^{2}$, S. Dembélé ${ }^{1}$, N. Le Fort-Piat ${ }^{1}$ \\ ${ }^{1}$ Femto-St Institute, UMR CNRS 6174 - UFC / ENSMM / UTBM. \\ Automatic Control and Micro-Mechatronic Systems Department. \\ 24 rue Alain Savary, 25000 Besançon, France \\ ${ }^{2}$ Université de Rennes 1, IRISA \\ INRIA Rennes-Bretagne Atlantique, Lagadic
}

Corresponding author: brahim.tamadazte@ens2m.fr

À paraître dans

The Int. Journal of Robotics Research, 2010

\begin{abstract}
This paper investigates sequential robotic microassembly for the construction of 3D micro-electro-mechanical systems (MEMS) structures using a 3D visual servoing approach. The previous solutions proposed in the literature for these kinds of problems are based on 2D visual control because of the lack of precise and robust 3D measures from the work scene. In this paper, the relevance of the real-time $3 \mathrm{D}$ visual tracking method and the 3D vision-based control law proposed is demonstrated. The 3D poses of the MEMS are supplied in real-time by a computer-aided design (CAD) model-based tracking algorithm. This latter is sufficiently accurate and robust to enable a precise regulation toward zero of the $3 \mathrm{D}$ error using the proposed pose-based visual servoing approach.

Experiments on a microrobotic setup have been carried out to achieve assemblies of two or more $400 \mu \mathrm{m} \times 400 \mu \mathrm{m} \times 100 \mu \mathrm{m}$ silicon micro-objects by their respective $97 \mu \mathrm{m} \times 97 \mu \mathrm{m} \times 100 \mu \mathrm{m}$ notches with an assembly clearance from $1 \mu \mathrm{m}$ to $5 \mu \mathrm{m}$. The different microassembly processes are performed with a mean error of $0.3 \mu \mathrm{m}$ in position and $0.35 \times 10^{-2} \mathrm{rad}$ in orientation.
\end{abstract}


keywords: MEMS, robotic microassembly, micromanipulation, pose-based visual control, virtual visual servoing, visual tracking.

\section{Introduction}

\subsection{Motivations}

There is a growing interest in 3D complex hybrid MEMS (Micro Electro Mechanical Systems), MOEMS (Micro Opto Electro Mechanical Systems) and BioMEMS (Biological MEMS) devices. They are used in a variety of domains as reported by (Wechsung, 2002): automotive, households, IT peripherals, bio-medical devices and telecommunications. The consequence of this strong demand is the emergence of an active research area concerning the development of assembly technologies on a microscale. A review of this research area had been carried out by (Cohn et al., 1998) and (Probst et al., 2009).

Hybrid MEMS that use different and incompatible materials or manufacturing processes as lithography, Deep Reactive Ion Etching (DRIE), Reactive Ion Etching (RIE) are produced by assembling individual microscale parts. Not only can microassembly combine such incompatible components but it can also increase the overall yield like the number of layers and manufacturing steps grow for a complex device. Microassembly can also overcome the planar, 2.5D shape limitations of standard MEMS manufacturing processes.

Microassembly approaches can be classified into self-assembly and robotic assembly. In the first case, processes in which a disordered system of pre-existing components forms an organized structure or pattern as a consequence of specific local interactions among the components themselves, without external direction (Zhou and Chang, 2006). In the second case, a robotic system combined with a microhandling system and an imaging system are used to reach the same objective (Popa and Stephanou, 2004). The robotic microassembly process can be divided into elementary tasks that are sequentially performed: visual detection of the component, positioning of the component, positioning of the end-effectors, grip of the component, transfer of the component, release of the component. The automation of MEMS assembly processes can vary between tele-operated, semi-automatic and fully automated assembly. Often, MEMS assembly tasks may require sub-micron level positioning accuracy that has to be achieved despite the unavoidable effects of thermal expansion or modeling and calibration errors in the manipulator system (del Corrala et al., 2002). In addition, while gravity is the dominant force in the macro domain, electrostatic forces, surface tension effects due to humidity and intermolecular van der Waals forces become dominant on the microscale, making it difficult to predict an object's behavior during manipulation (Cohn et al., 1998). Unlike the conventional robotic assembly, operations in the macroworld which rely on accurate robots that play back recorded motions. However, this form of open-loop manipulation is not suitable to the microscale due to the increased precision requirements and the vastly different mechanics of manipulation. So, the closed-loop is a good solution to automate MEMS assembly processes essentially using vision-based control mechanisms (Yesin and Nelson, 2005), (Abbott et al., 2007). Among works relative to closed-loop strate- 
gies based on vision feedback, we can cite those of (Feddema and Simon, 1998), (Fatikow et al., (1999), (Ralis et al., 2000), (Yang et al., 2003), (Popa and Stephanou, 2004), (Yesin and Nelson, 2005). Vision feedback control is sometimes combined with force feedback, as in the works of (Nelson et al., 1999), (Chen et al., 2004) and (Greminger and Nelson, 2004). This hybrid vision/force feedback approach is essentially used to automate the micro-object grip task.

\subsection{Previous research in MEMS assembly}

Vision-based control mechanisms in microhandling and microassembly tasks require high magnification and high resolution imaging systems. These imaging systems often consist of optical microscopes. Nevertheless, such imaging systems have limitations of the field-of-view and depth-of-field. Thus, a large part of microassembly workcells presented in literature is equipped with distributed vision systems (two or more optical microscopes and conventional CCD cameras). Therefore, high resolution images (for local viewing) can be combined with low resolution images (for global viewing). Indeed, high resolution images lead to precise measurements but very small field-of-view, like low resolution images enable large field-ofview but less precise information (Ralis et al., 2000), (Tao et al., 2005). To overcome this duality, two kinds of solutions had been implemented: a switch between the components of a multiple view system (Yang et al., 2003), (Popa and Stephanou, 2004), (Sun and Chin, 2004), (Abbott et al., 2007), (Probst et al., 2009), or integration of a dynamic zoom control in the visual control (Tao et al., 2005), (Tamadazte et al., 2008b). The other advantage of using a distributed vision system is the ability to have access to the depth of the scene using lateral views (Yang et al., 2005). Manipulations are often performed in conjunction with devices such as optical microscopes, scanning electron microscopes (SEM) or transmission electron microscopes (TEM), which all provide sensory feedback in the form of a monocular image. The SEM is often favoured for nanomanipulation owing to its high resolution and depth-of-field (Fatikow et al., 2009), (Jasper and Fatikow, 2009), (Kratochvil et al., 2009).

The guidance of robots through real-time and continuous visual feedback is generally known as visual servoing, and the continuous observation of the objects of interest is referred to visual tracking. Therefore, in literature, most of the researchers focus on developing tracking techniques (Feddema and Simon, 1998), (Greminger and Nelson, 2005) and visionbased control approaches (Zhou and Nelson, 1999), (Ralis et al., 2000), (Sun and Chin, 2004) dedicated to the microscale applications. Some authors have investigated other visual techniques which consist in using virtual reality to assist the human operator working in teleoperated mode or to directly use these techniques to automate MEMS assembly and handling tasks using vision feedback control (Kawaji and Fukuda, 2001), (Ammi and Ferreira, 2007). As regards the most studied microassembly tasks, we find the positioning, the grip and the insertion (such as peg-in-hole) tasks (Yang et al., 2003), (Popa and Stephanou, 2004), (Tao et al., 2005), (Tamadazte et al., 2009). 


\subsection{Contributions and organization}

As a complement to this intensive research, we investigate 3D vision-based control mechanisms in order to automate the robotic assembly of 3D multi-part structures. These methods are tested on the assembly of $400 \mu \mathrm{m} \times 400 \mu \mathrm{m} \times 100 \mu \mathrm{m}$ (length, with and depth of the micropart, respectively) planar silicon microparts. The considered application consists of the assembly of two types of MEMS i.e. simple MEMS and complex MEMS. The first one is obtained by the assembly of two microparts using their respective $97 \mu \mathrm{m} \times 97 \mu \mathrm{m} \times 100$ $\mu \mathrm{m}$ notches and in the second one, five of the same microparts are assembled on three levels, also using their notches.

To track the microscale parts to be assembled over frames during the experiment, a 3D model based tracking algorithm is developed. This algorithm is able to directly provide in real time and using a single view of the scene, the 3D pose of one single micropart (single tracking) or of several microparts at the same time (multiple tracking). Firstly, the main advantage of a model based method is that the knowledge of the scene (the implicit 3D information) allows robustness and performance to be improved by predicting hidden movement of the objects and reducing the effects of outliers data introduced in the tracking process. Secondly, the traditional multi-view imaging system which equipped much of the microassembly workcells is replaced by a mono-view system. The efficiency of this algorithm is also demonstrated by tracking different objects under various imaging systems. Thus, a $80 \mathrm{~mm} \times 80 \mathrm{~mm} \times 10 \mathrm{~mm}$ macroscale part is tracked using a pin-hole camera equipped with a conventional objective lens, a $400 \mu \mathrm{m} \times 400 \mu \mathrm{m} \times 100 \mu \mathrm{m}$ under an optical microscope and a $40 \mu \mathrm{m} \times 40 \mu \mathrm{m} \times 5 \mu \mathrm{m}$ inside a SEM.

The experimental validation of the combination of the CAD model based visual tracking and the pose-based visual control approaches provide a high repeatability and precision during the several assembly processes achieved. Thus, for a series of 10 successive assemblies performed, a $100 \%$ success rate is obtained with a mean precision of $0.3 \mu \mathrm{m}$ in position and $0.35 \times 10^{-2} \mathrm{rad}$ in orientation. Concerning the mean cycle time necessary to assemble two microparts (including planar positioning of the first micropart, spatial positioning of the second micropart and the insertion of the two microparts) is about 40.8 seconds. Such precise assembly in real-time has never been presented in literature.

This paper is structured as followed: Section II gives the specification of the task to be carried out as well as the considered microrobotic system. Section III presents the 3D CAD model based tracking algorithm used to track the microparts to assemble. This algorithm is also tested on different sequences of images acquired with various imaging systems: CCD camera equipped with a conventional objective lens (low magnification), optical microscope (high magnification) and SEM (very high magnification). Section IV describes the developed pose-based visual control law. Section V presents experimental results of the multiple microparts insertions. The techniques developed in this paper are also validated on a fullyautomatic microassembly of five microparts to build a more complex 3D MEMS on three levels. Discussions about possible future works, particularly the way to decrease the assembly cycle time, to increase the number of microparts to assemble and concentrate efforts on 
the assembly of more complex MEMS, will be considered.

\section{Task specification and microsassembly workcell}

To validate the vision algorithms and control concepts proposed, a microassembly workcell, illustrated in Fig. 3, had been developed in our laboratory. It includes a robotic system with five high accuracy degrees-of-freedom (DOF), a microhandling system and an imaging system, everything being placed in a controlled environment. This workcell is shown in the Extension 6 (refer to the Appendix A for details).

\subsection{Manipulated objects}

The development of microtechnologies enables the manufacturing of microscale parts by etching fine structures in silicon wafers. These microparts are obtained using a DRIE process. Several micro-objects with different shapes and sizes are realized. Notches are etched into two or four sides of each micropart. Figure 1 shows the CAD model and the corresponding total dimensions of some samples.

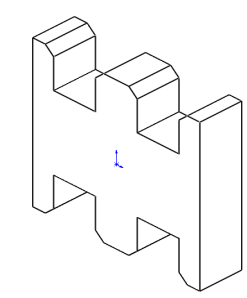

$400 \times 400 \times 100 \mu \mathrm{m}^{3}$ [heigth, width, depth]

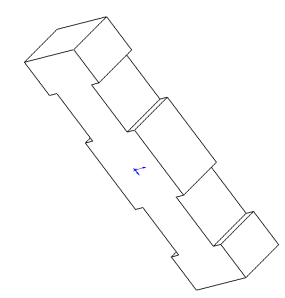

$400 \times 100 \times 100 \mu \mathrm{m}$

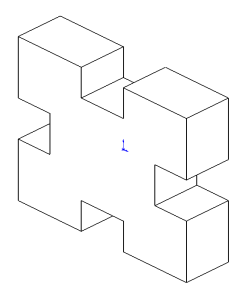

$400 \times 400 \times 100 \mu \mathrm{m}^{3}$

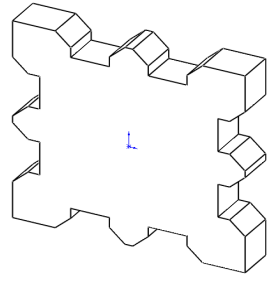

$600 \times 600 \times 100 \mu \mathrm{m}^{3}$

Figure 1: Examples of CAD models of MEMS microstructures.

This paper targets the assembly process by fitting together two or more microparts of dimensions $400 \mu \mathrm{m} \times 400 \mu \mathrm{m} \times 100 \mu \mathrm{m}$ into their respective $97 \mu \mathrm{m} \times 97 \mu \mathrm{m} \times 100 \mu \mathrm{m}$ notches (square holes). It can be noted that both i.e. microparts and notches are etched with a precision of $\pm 1 \mu \mathrm{m}$. Therefore, the assembly clearance of two microparts ranges between $1 \mu \mathrm{m}$ (minimum assembly clearance) and $5 \mu \mathrm{m}$ (maximum assembly clearance).

Let $\mathbf{A}$ and $\mathbf{B}$ be the microparts to assemble (Fig. 2), the objective is then to automatically insert a notch of $\mathbf{A}$ into a notch of $\mathbf{B}$.

This problem can be divided into three basic tasks that should be performed in a suitable sequence: the positioning of the micropart $\mathbf{A}($ task\#1), the positioning of the micropart $\mathbf{B}$ (task\#2) at its insertion position and the vertical insertion of $\mathbf{B}$ into $\mathbf{A}$ (task\#3), see Fig. 2. 


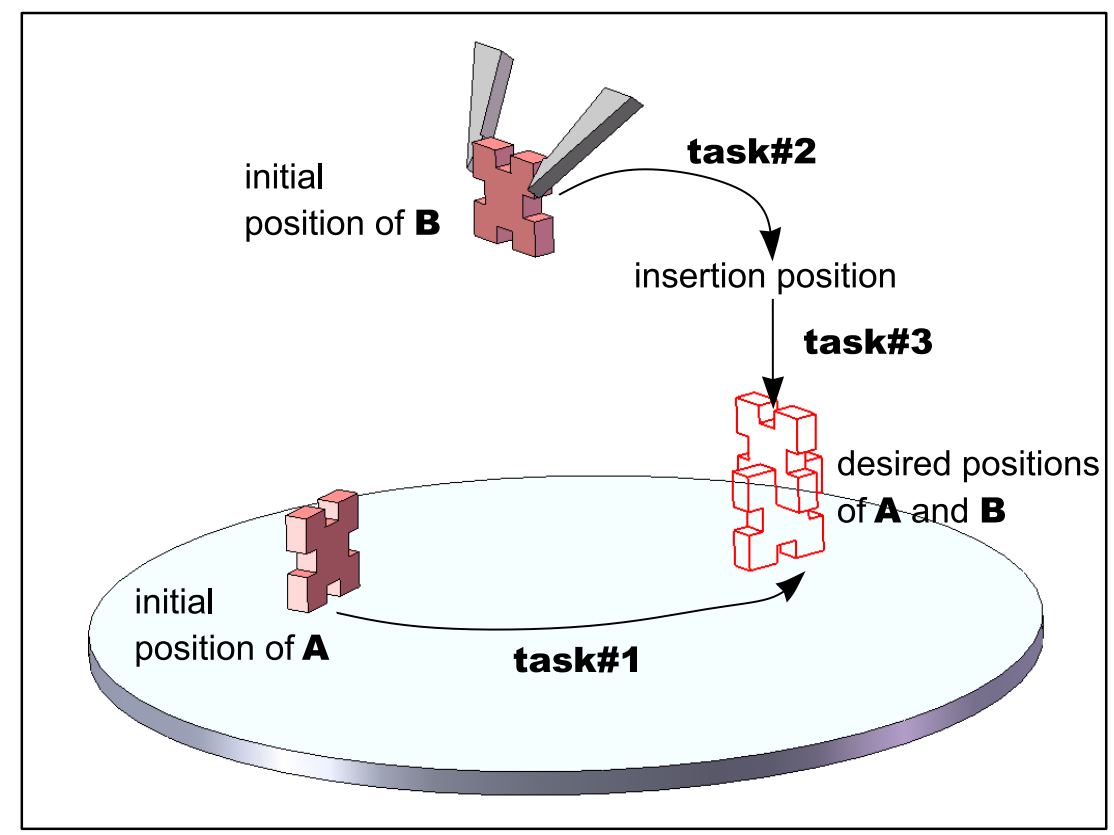

Figure 2: Tasks description: task\#1, task\#2 and task\#3 correspond to the positioning of the micropart $\mathbf{A}$, the positioning of the micropart $\mathbf{B}$ and the insertion of $\mathbf{B}$ into $\mathbf{A}$, respectively.

Let us note that this paper describes the microassembly of two microparts, but as is shown in the results section. The proposed methodologies are also tested to assemble more complex assemblies.

\subsection{Microrobotic workstation}

The complete workstation includes a robotic system in combination with a microhandling and an imaging systems. The whole setup is located inside a controlled environment and posed on a vibrations-free table (Fig. 3). Two PCs connected by an Ethernet link processes the information, the first (Pentium (R) D, CPU $2.80 \mathrm{G} \mathrm{Hz}, 2$ Go of RAM) is dedicated to vision algorithms while the second (Pentium (R) 4, CPU $3.00 \mathrm{G} \mathrm{Hz}$, and 1 Go of RAM) is used for control algorithms.

From a kinematic point of view, the workstation is a five DOF robotic system (Fig. 4). Three DOF in translation are achieved by three high accuracy linear stages and two DOF in rotation are achieved by two high accuracy angular stages (all from Polytec PI). The features of these stages are summarized in Table 1. The five DOF are distributed into two robotic systems: a $x y \alpha$ system and a $z \varphi$ system. The former system (the positioning platform) is equipped with a compliant table (the table is supported by three springs) and enables the positioning in the horizontal plane. The latter system (the manipulator) supports the gripper and enables the vertical positioning and spatial orientation of microparts. 

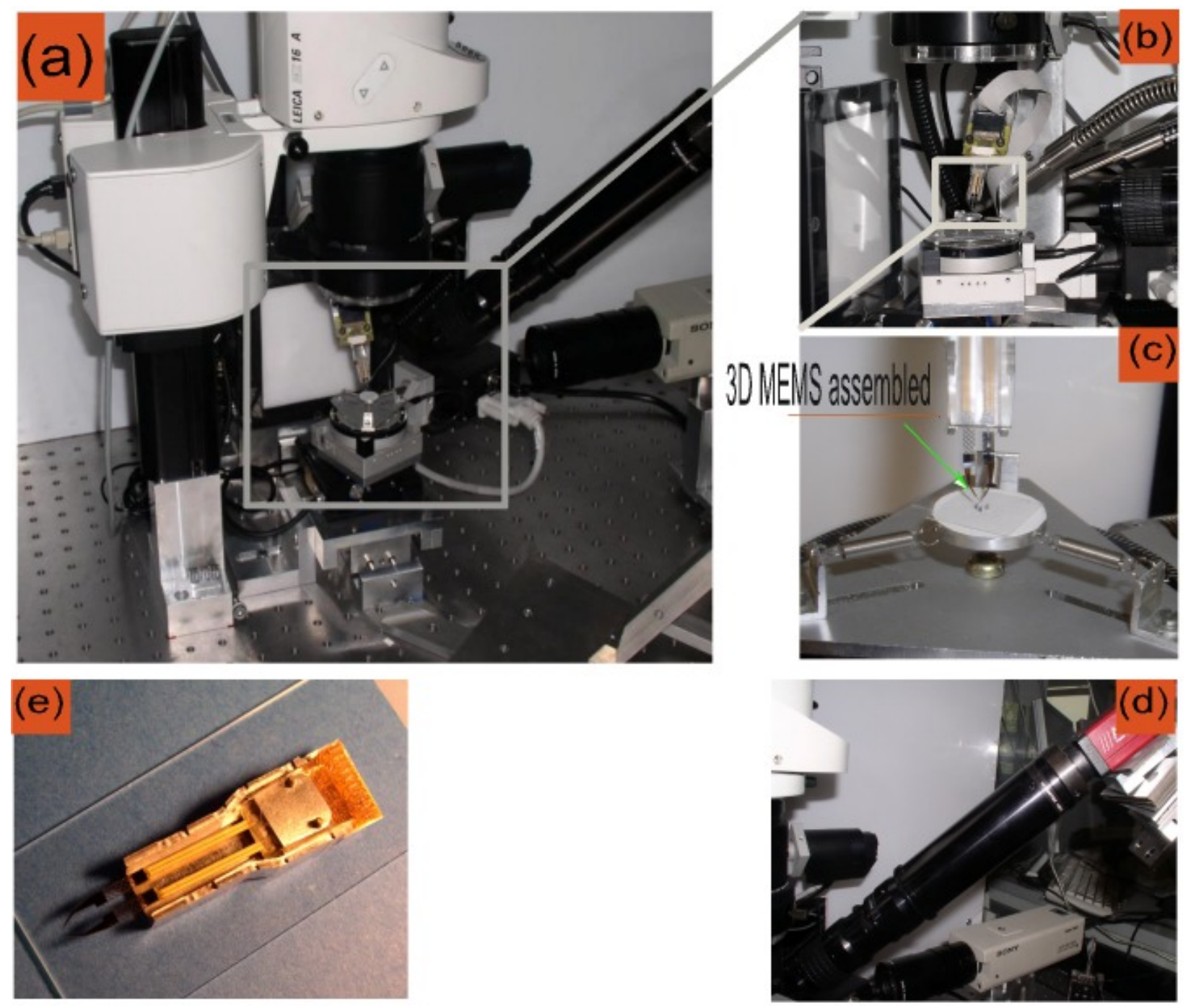

Figure 3: Image (a): the global view of the workcell positioned inside a controlled environment and on a vibration-free table, image (b): zoom on the robotic system (3 DOF positioning platform and 2 DOF manipulator), image (c): zoom on the compliant support and the gripper, image (d): zoom on the imaging system and image (e): zoom on the 4 DOF microgripper with nickel end-effectors.

\subsubsection{Microhandling system}

A Microrobotic Microprehensile On Chip (MMOC) gripper (Fig. 3.e) developed in the department AS2M of FEMTO-ST Institute is used for the handling of micro-objects (Agnus et al., 2005). It is a two-fingered microhandling system with four DOF (two DOF by finger). It enables open-and-close motions as well as small up-and-down motions. Every finger is a piezoelectric bimorph with an end-effector made of silicon layers (12 $\mu \mathrm{m}$ and $400 \mu \mathrm{m})$ 
Table 1: Linear and angular stages specifications.

\begin{tabular}{l|l}
\hline \multicolumn{2}{c}{ Linear motion stages } \\
\hline resolution & $0.007 \mu \mathrm{m}$ \\
increment & $0.05 \mu \mathrm{m}$ \\
max velocity & $1.5 \mathrm{~mm} / \mathrm{s}$ \\
stroke & $25 \mathrm{~mm}$ \\
\hline \hline \multicolumn{2}{c}{ Angular motion stages } \\
\hline resolution & $26 \mu \mathrm{rad}$ \\
increment & $26 \mu \mathrm{rad}$ \\
max velocity & $0.78 \mathrm{rad} / \mathrm{s}$ \\
\hline
\end{tabular}

separated by an oxide layer $(1 \mu \mathrm{m})$.

Modularity is an important design criterion during development, so the MMOC microgripper has been designed in order to use different end-effectors (finger tips). Thus, it can grab a high variety of objects according to the type of end-effectors used: planar silicon microparts, balls, gears, optical fibers, etc. The specimen used in the current experiments are endowed with nickel end-effectors. The corresponding characteristics and performances

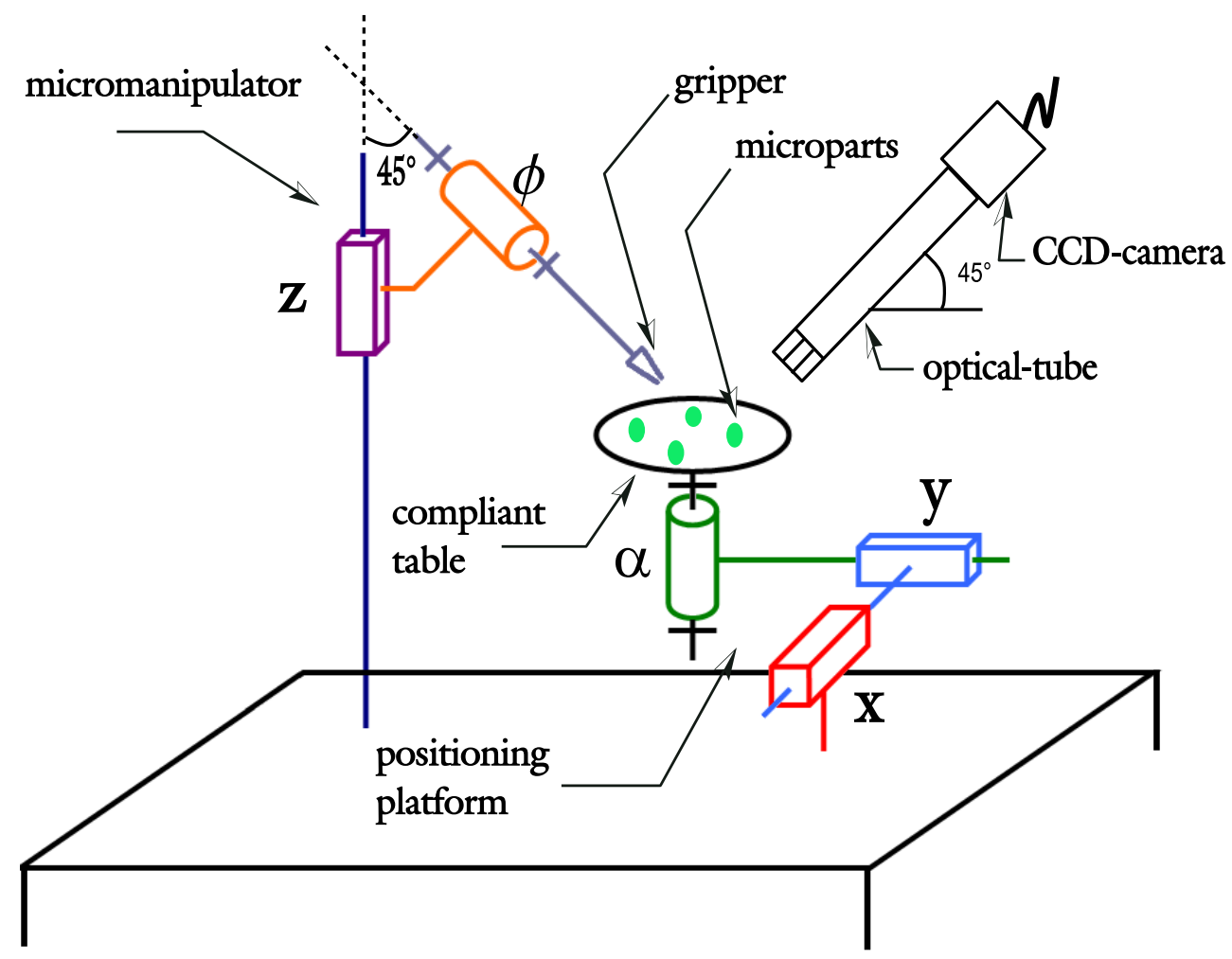

Figure 4: Mechanical structure of the microassembly workcell. 
are summarized in Table 2.

Table 2: Specifications of the nickel end-effectors gripper.

\begin{tabular}{l|l}
\hline \multicolumn{2}{c}{ Typical strokes } \\
\hline open-close & $320 \mu \mathrm{m}$ \\
up-and-down & $200 \mu \mathrm{m}$ \\
\hline \hline \multicolumn{2}{c}{ Blocking forces } \\
\hline open-close & $55 \mathrm{mN}$ \\
up-and-down & $10 \mathrm{mN}$ \\
\hline \hline \multicolumn{2}{c}{ Other characteristics } \\
\hline High resolution & $\sim 10 \mathrm{~nm}$ \\
\hline Speed & $\prec 10 \mathrm{~ms}$ \\
\hline
\end{tabular}

\subsubsection{Imaging system}

The imaging system comprises two photon videomicroscopes. The first optical microscope of LEICA MZ16A type delivers vertical images of the scene. However, this microscope is not used in the current experiments (but used for 2D multiple scale image-based visual servoing). The second is a CCD camera associated with a $11.452 \mathrm{~mm}$ focal-length lens and a $140 \mathrm{~mm}$ optical tube. It is positioned at an angle of $\pi / 4 \mathrm{rad}$ from the horizontal plane in order to ensure the best perspective view during the assembly tasks. The image format is $1280 \times$ 960 pixels. The other specifications include: a resolution of $0.95 \mu \mathrm{m}$, a working distance of $80 \mathrm{~mm}$, a field-of-view of $1.216 \mathrm{~mm} \times 0.912 \mathrm{~mm}$ and a depth-of-field which varies between $0.09 \mathrm{~mm}$ to $0.98 \mathrm{~mm}$ with respect to the magnification value.

According to (Zhou and Nelson, 1999), (Ammi et al., 2005), (Figl et al., 2005) and (Tamadazte et al., 2008a) the latter videomicroscope can be described by the linear perspective model whose parameters are the scale factor, the focal-length, the position of the principal point. The focal-length parameter $(f)$ is the sum of the lens focal-length $\left(f_{0}\right)$ with the optical tube-length $\left(t_{0}\right)$. A $2 \mathrm{D}$ calibration template is used to identify these parameters.

\subsection{Environment control system}

Unlike in macroscale conditions, small changes in temperature, humidity or cleanliness (presence of external microparticles) induce large effects on the microscale. The adhesion forces (electrostatic, van der Waals, etc.) and the microtribological proprieties of microparts are strongly affected. (del Corrala et al., 2002) performed two tests of pick-and-place operations. In the first test, the temperature is constant $\left(25^{\circ} \mathrm{C}\right)$ and the humidity ranged from $10 \%$ of humidity rate (HR) to $80 \% \mathrm{HR}$ by step of $10 \% \mathrm{HR}$. In the second test, the humidity is constant but the temperature ranged between $0^{\circ} \mathrm{C}$ and $40^{\circ} \mathrm{C}$ with $5^{\circ} \mathrm{C}$ increment. The pick-and-place operations showed important translation and rotation errors according to the 
temperature and humidity conditions. For other reasons such as dust-microparticle avoidance on the manipulation area, it is strongly recommended to use a controlled environment (del Corrala et al., 2002), (Zhou et al., 2002) and (Devanathan et al., 2006). Therefore, the current experiments took place in a controlled environment and on a vibrations-free table, providing maximum rejection of environmental perturbations.

\section{CAD model based tracking algorithm}

To be able to control the micromanipulator, it is necessary to track over frames the microscale object to be manipulated during the experiment. As shown in the next section, since we considered a 3D control and we decided to use a 3D model based tracker that is able to directly provide the $3 \mathrm{D}$ object pose ${ }^{c} \mathbf{M}_{A}$ (resp. ${ }^{c} \mathbf{M}_{B}$ ) using a single view of the scene. ${ }^{c} \mathbf{M}_{A}$ represents the $3 \mathrm{D}$ pose of the micropart $\mathbf{A}$ in the camera frame $\mathcal{R}_{c}$. A complete definition of these 3D transformations is given in Appendix A. These techniques have applications in many domains ranging from augmented reality to visual servoing and even medical imaging or industrial applications. The main advantages of a model based method are that the knowledge about the scene (the implicit 3D information) allows improvements of the robustness and performances by being able to predict hidden movement of the object and acts to reduce the effects of outliers data introduced in the tracking process. Considering microsystems, such trackers have already been considered (eg in (Yesin and Nelson, 2005), (Stoeter et al., 2006), (Kratochvil et al., 2009)).

The approach proposed in (Drummond and Cipolla, 2002), (Comport et al., 2006) rely on full-scale non-linear optimization techniques which consist in minimizing the error between the observation and the forward-projection of the model (Haralick et al., 1989), (Lowe, 1991). This approach consists in estimating the real camera pose ${ }^{c} \mathbf{M}_{o}$ or displacement by minimizing the error $\Delta$ between the observed data $\mathbf{s}^{*}$ and the current value $\mathbf{s}(\mathbf{r})$ of the same features computed using the model according to the current pose $\mathbf{r}$ (which is a vector-based representation of the pose ${ }^{c} \mathbf{M}_{o}$, see Appendix A for details):

$$
\Delta=\sum_{i=1}^{N}\left(s_{i}(\mathbf{r})-s_{i}^{*}\right)^{2},
$$

Minimization is handled using numerical iterative algorithms such as Newton-Raphson or Levenberg-Marquardt. Assuming that the low level data extracted from the image are likely to be corrupted, we use a statistically robust camera pose estimation process (based on the widely accepted statistical techniques of robust M-estimation (Huber, 1981)). The goal is to minimize

$$
\Delta_{\mathcal{R}}=\sum_{i=1}^{N} \rho\left(s_{i}(\mathbf{r})-s_{i}^{*}\right),
$$

where $\rho(u)$ is a robust function (Huber, 1981) introduced in the objective function in order to reduce the sensitivity to outliers. Iteratively Re-weighted Least Squares (IRLS) is a 
common method for applying the M-estimator. It converts the M-estimation problem into an equivalent weighted least-squares problem.

In this paper, this full scale non-linear optimization is formulated in terms of a Virtual Visual Servoing problem (Comport et al., 2006). This method considers the 2D visual servoing framework for controlling the motion of a virtual camera so that the projection in the image of the object model perfectly fits with the current position of the object in the image acquired by the real camera. We thus obtain a virtual feature-based system which is able to treat complex scenes in real-time without the need of markers on the objects. The error $\Delta$ to be regulated to 0 is then defined as:

$$
\Delta_{\mathcal{R}}=\mathbf{D}\left(\mathbf{s}(\mathbf{r})-\mathbf{s}^{*}\right),
$$

where $\mathbf{D}$ is a diagonal weighting matrix given by $\mathbf{D}=\operatorname{diag}\left(w_{1}, \ldots, w_{k}\right)$. The weights $w_{i}$ reflect the confidence in each feature and their computation is based on M-estimators and is described in (Drummond and Cipolla, 2002) and (Comport et al., 2006).

A virtual camera, defined by its position $\mathbf{r}$ in the object frame can be virtually moved in order to minimize this error. At convergence the position of the virtual camera will be aligned with the real camera pose. This can be achieved by considering a simple control law given by:

$$
\mathbf{v}=-\lambda\left(\mathbf{D L}_{\mathbf{s}}\right)^{+} \mathbf{D}\left(\mathbf{s}(\mathbf{r})-\mathbf{s}^{*}\right),
$$

where $\mathbf{v}$ is the velocity screw of the virtual camera and $\mathbf{L}_{\mathbf{s}}$ is the interaction matrix or image Jacobian related to $\mathbf{s}$ and defined such as $\dot{\mathbf{s}}=\mathbf{L}_{\mathbf{s}} \mathbf{v}$. Rodrigues' formula is then used to map the velocity vector $\mathbf{v}$ to its corresponding instantaneous displacement allowing the pose to be updated. To apply the update to the displacement between the object and camera, the exponential map is applied using homogeneous matrices ${ }^{c} \mathbf{M}_{o}$ that describe the pose resulting in:

$$
{ }^{c} \mathbf{M}_{o}^{t+1}={ }^{c} \mathbf{M}_{o}^{t} e^{\mathbf{v}}
$$

where $t$ denotes the number of iterations of the minimization process.

In this paper, we consider features corresponding to a distance between the forwardprojection of the contours of a CAD model (see Figure 1) and local point features obtained from a 1D search along the normal to the contour. More precisely if we denote $\mathbf{p}_{i}, i=1 . . k$ these points and $\mathbf{l}_{i}(\mathbf{r})$ the projection of the corresponding line for the pose $\mathbf{r}$, the elements of vector $\mathbf{s}$ used in equation (1) can be defined by:

$$
s_{i}(\mathbf{r})=d_{\perp}\left(\mathbf{p}_{i}, \mathbf{l}_{i}(\mathbf{r})\right),
$$

where $d_{\perp}(\mathbf{p}, \mathbf{l}(\mathbf{r}))$ is the orthogonal distance between $\mathbf{p}$ and $\mathbf{l}(\mathbf{r})$. The distance $d_{\perp}($.$) is$ represented on Fig. 5. For such a distance feature, we have $s_{i}^{*}=0$. The interaction (or Jacobian) used in equation (4) has to be defined and is given in (Comport et al., 2006). In this case the desired values of these distances are equal to zero. The assumption is made that the contours of the object in the image can be described as piecewise linear segments. All distances are then treated according to their corresponding segment. An example of the application of the tracking algorithm to a square silicon micropart is shown in Fig. 6. 


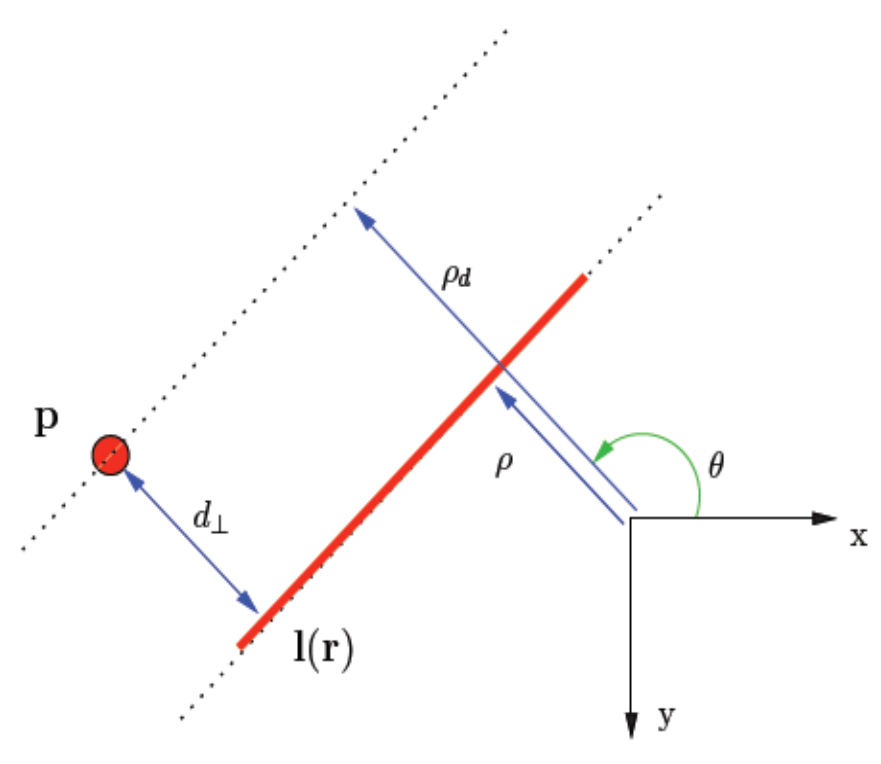

Figure 5: Distance of a point to a line.

\section{Assembly on the microscale by means of 3D visual control}

Although visual servoing has been widely considered in microassembly (Yesin and Nelson, 2004), (Feddema and Simon, 1998), (Chen et al., 2004), and (Tamadazte et al., 2008b), most of systems consider image-based visual servoing (IBVS) approach rather than position-based visual servoing (PBVS). Refer to (Hutchinson et al., 1996), (Chaumette and Hutchinson, 2006), (Chaumette and Hutchinson, 2007) for a very good description of the IBVS and the PBVS. At the macroscale level, the positioning of a camera supported by a robot with respect to a target using 3D visual servo is widely considered (Wilson et al., 1996), (Martinet and Gallice, 1999). However, previous few publications (Yesin and Nelson, 2004) have investigated the assembly of microparts by implementing 3D visual control on the microscale.

The main advantage is that the task is described as the regulation to zero of a $3 \mathrm{D}$ error between the positions of the two microparts. The use of a tracker that provides microparts 3D localization, can be regarded as a drawback, but in fact it provides a more robust method for tracking object in the image sequence, as will be seen in the next section.

\subsection{Notations}

Let $\mathcal{R}_{c}, \mathcal{R}_{A}, \mathcal{R}_{A^{*}}, \mathcal{R}_{B}$ and $\mathcal{R}_{B_{2}^{*}}$ be the frame attached to the camera (i.e. the videomicroscope), the current and final frames of the micropart $\mathbf{A}$, the current and final frames of the micropart $\mathbf{B}$, respectively. Moreover, an insertion frame $\left(\mathcal{R}_{B_{1}^{*}}\right)$ for $\mathbf{B}$ is required where the part process through before switching to the insertion stages.

The tracking algorithm provides, for each new image, the following information: 


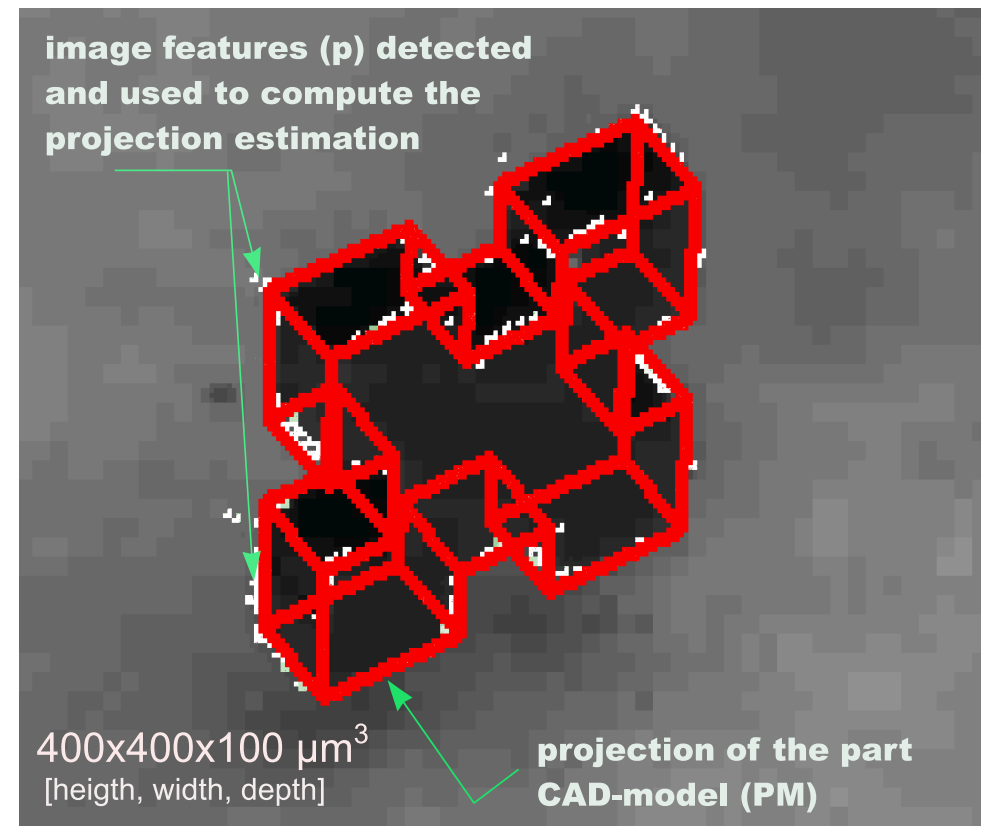

Figure 6: Application of the tracker on a micropart. The points (p) are used to estimate the CAD model image projection (PM) as at is shown in the image.

- ${ }^{c} \mathbf{M}_{A}$, the homogeneous transformation between the camera frame and the current position of $\mathbf{A}$,

- ${ }^{c} \mathbf{M}_{B}$, the homogeneous transformation between the camera frame and the current frame of $\mathbf{B}$.

Let

- ${ }^{c} \mathbf{M}_{A^{*}}$ be the homogeneous transformation between the camera frame and the desired frame of $\mathbf{A}$,

- ${ }^{c} \mathbf{M}_{B_{1}^{*}}$ be the homogeneous transformation between the camera frame and the insertion frame of $\mathbf{B}$,

- ${ }^{c} \mathbf{M}_{B_{2}^{*}}$ be the homogeneous transformation between the camera frame and the desired frame of $\mathbf{B}$.

\subsection{Control law}

Let us decompose the insertion task into three different tasks (assembly subtasks):

1. task\#1: displacement of the micropart $\mathbf{A}$ to a given position (defined by $\mathcal{R}_{A^{*}}$ ); the achievement of this task is ensured by a control law regulating to zero the error defined 


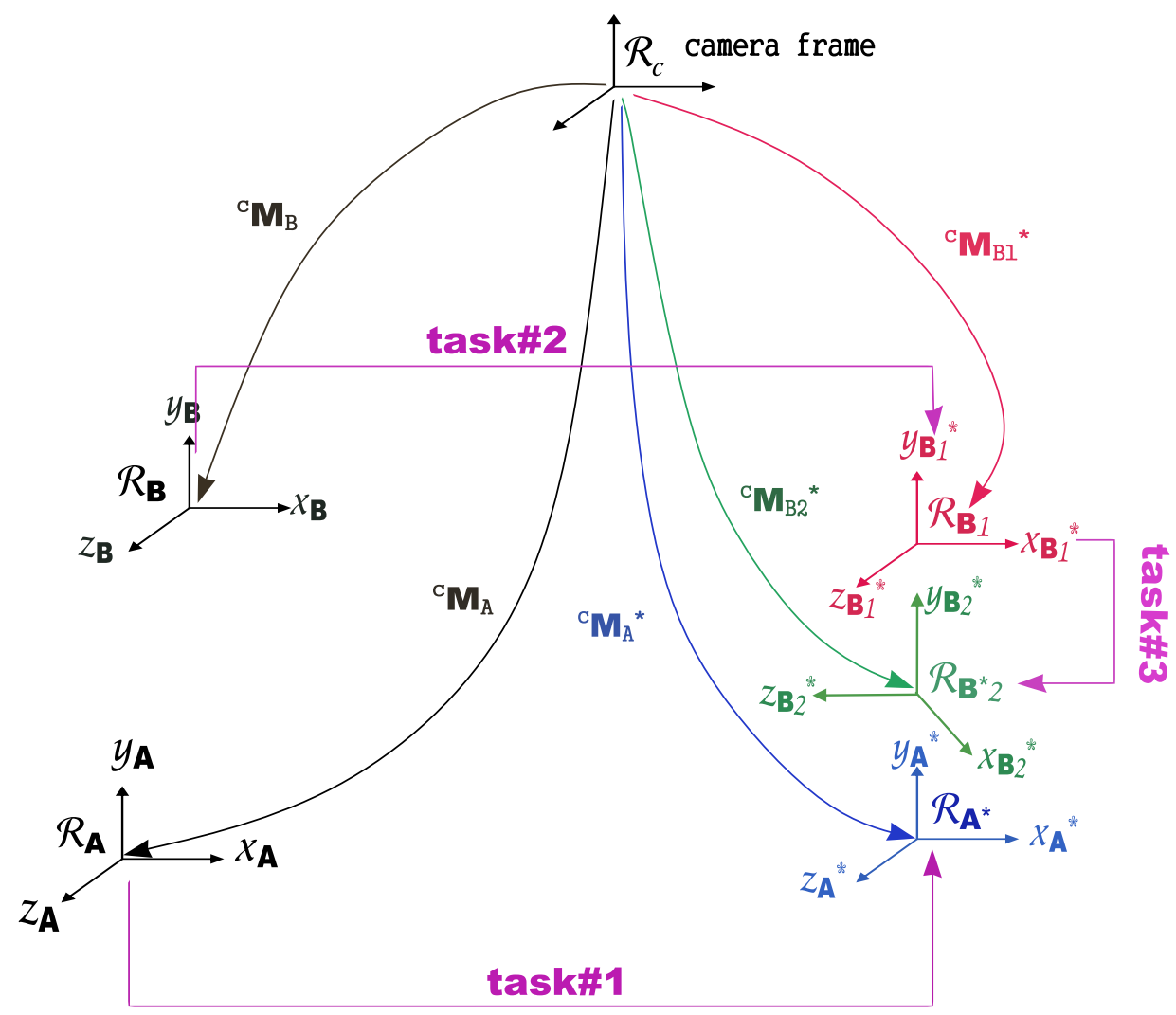

Figure 7: Methodology of the insertion of the micropart $\mathbf{B}$ into the micropart $\mathbf{A}$.

as:

$$
{ }^{A} \mathbf{M}_{A^{*}}={ }^{c} \mathbf{M}_{A}^{-1}{ }^{c} \mathbf{M}_{A^{*}}
$$

2. task\#2: displacement of the second micropart $\mathbf{B}$ to an intermediate position (defined by $\left.\mathcal{R}_{B_{1}^{*}}\right)$. the achievement of this task is ensured by a control law regulating to zero the error defined as:

$$
{ }^{B} \mathbf{M}_{B_{1}^{*}}={ }^{c} \mathbf{M}_{B}^{-1} \quad{ }^{c} \mathbf{M}_{B_{1}^{*}}
$$

3. task\#3: insertion of micropart $\mathbf{B}$ in micropart $\mathbf{A}$ (defined by $\mathcal{R}_{B_{2}^{*}}$ ). the achievement of this task is ensured by a control law regulating to zero the error defined as:

$$
{ }^{B} \mathbf{M}_{B_{2}^{*}}={ }^{c} \mathbf{M}_{B}^{-1}{ }^{c} \mathbf{M}_{B_{2}^{*}}
$$

Switching between task\#2 and task\#3 may be done when the error ${ }^{B} \mathbf{M}_{B_{1}^{*}}$ is small enough.

As in most of visual servoings, a key problem is the definition of the object desired position in the image. In our case we have to determine ${ }^{c} \mathbf{M}_{A^{*}},{ }^{c} \mathbf{M}_{B_{1}^{*}},{ }^{c} \mathbf{M}_{B_{2}^{*}}$. These microparts desired 
positions are performed in tele-operated mode (using a joystick) and the corresponding poses are recorded. The precise definition of $\mathcal{R}_{B_{1}}$ is important for the success of the insertion (Fig. 7). Another solution using directly the CAD model of the micropart will be investigated in the near future.

Let us define $\mathcal{R}_{\mathcal{F}}$ the base frame of the workcell. The homogeneous transformation between the camera frame $\mathcal{R}_{c}$ and the workcell frame $\mathcal{R}_{\mathcal{F}}$ is noted ${ }^{\mathcal{F}} \mathbf{M}_{A}$ and can be computed as:

$$
{ }^{\mathcal{F}} \mathbf{M}_{A}={ }^{\mathcal{F}} \mathbf{M}_{c}{ }^{c} \mathbf{M}_{A}
$$

where ${ }^{\mathcal{F}} \mathbf{M}_{c}$ is the position of the camera in the reference frame of the workcell (which is a known constant) and ${ }^{c} \mathbf{M}_{A}$ is given by the tracking algorithm. Therefore, the $3 \mathrm{D}$ current poses ${ }^{c} \mathbf{M}_{A},{ }^{c} \mathbf{M}_{B}$ defined in the camera frame $\mathcal{R}_{c}$ can be represented in the workcell frame $\mathcal{R}_{\mathcal{F}}$ by:

$$
\begin{aligned}
{ }^{\mathcal{F}} \mathbf{M}_{A} & ={ }^{\mathcal{F}} \mathbf{M}_{c}{ }^{c} \mathbf{M}_{A} \\
{ }^{\mathcal{F}} \mathbf{M}_{B} & ={ }^{\mathcal{F}} \mathbf{M}_{c}{ }^{c} \mathbf{M}_{B}
\end{aligned}
$$

It is the same for ${ }^{c} \mathbf{M}_{A^{*}},{ }^{c} \mathbf{M}_{B_{1}^{*}}$ and ${ }^{c} \mathbf{M}_{B_{2}^{*}}$,

$$
\begin{gathered}
{ }^{\mathcal{F}} \mathbf{M}_{A_{1}^{*}}={ }^{\mathcal{F}} \mathbf{M}_{c}{ }^{c} \mathbf{M}_{A_{1}^{*}} \\
{ }^{\mathcal{F}} \mathbf{M}_{B_{1}^{*}}={ }^{\mathcal{F}} \mathbf{M}_{c}{ }^{c} \mathbf{M}_{B_{1}^{*}} \\
{ }^{\mathcal{F}} \mathbf{M}_{B_{2}^{*}}={ }^{\mathcal{F}} \mathbf{M}_{c}{ }^{c} \mathbf{M}_{B_{2}^{*}}
\end{gathered}
$$

For instance, to regulate to zero the error defined from ${ }^{A} \mathbf{M}_{A^{*}}$ a solution consists of choosing $\mathbf{s}=\left({ }^{\mathcal{F}} \mathbf{t}_{A}, \theta \mathbf{u}\right)$ as the current 3D pose, $\mathbf{s}^{*}=\left({ }^{\mathcal{F}} \mathbf{t}_{A^{*}}, \mathbf{0}\right)$ as the desired pose of the micropart $\mathbf{A}$, respectively. In this case, the error ${ }^{A} \mathbf{M}_{A^{*}}$ can be represented by:

$$
\mathbf{e}=\left({ }^{\mathcal{F}} \mathbf{t}_{A}-{ }^{\mathcal{F}} \mathbf{t}_{A^{*}}, \theta \mathbf{u}\right)
$$

The equation that links the time variation $\dot{\mathbf{s}}=\left(\dot{\mathcal{F}}_{A}, \dot{\theta \mathbf{u}}\right)^{\top}$ of $\mathbf{s}$ to the robot velocity in the workcell reference frame are given in (Marchand et al., 2002) by:

$$
\left(\begin{array}{c}
\mathcal{F}^{\dot{\mathbf{t}}_{A}} \\
\dot{\theta \mathbf{u}}
\end{array}\right)=\left(\begin{array}{cc}
\mathbf{I}_{3 \times 3} & \mathbf{0}_{3 \times 3} \\
\mathbf{0}_{3 \times 3} & \mathbf{J}_{\omega}
\end{array}\right)\left(\begin{array}{c}
\boldsymbol{v} \\
\boldsymbol{\omega}
\end{array}\right)_{\mathcal{F}}
$$

where

$$
\mathbf{J}_{\omega}=\mathbf{L}_{\omega}{ }^{c} \mathbf{R}_{\mathcal{F}}
$$

and

$$
(\boldsymbol{v}, \boldsymbol{\omega})_{\mathcal{F}}^{\top}=\left(\boldsymbol{v}_{x}, \boldsymbol{v}_{y}, \boldsymbol{v}_{z}, \boldsymbol{\omega}_{\alpha}, \boldsymbol{\omega}_{\beta}, \boldsymbol{\omega}_{\varphi}\right)_{\mathcal{F}}^{\top}
$$


According to (Malis et al., 1999), $\mathbf{L}_{\omega}$ is such that:

$$
\mathbf{L}_{\omega}^{-1} \theta \mathbf{u}=\theta \mathbf{u}
$$

Using a 6 DOF robot, we would obtain the following control law:

$$
\begin{aligned}
\left(\begin{array}{c}
\boldsymbol{v} \\
\boldsymbol{\omega}
\end{array}\right)_{\mathcal{F}} & =-\lambda\left(\begin{array}{cc}
\mathbf{I}_{3 \times 3} & \mathbf{0}_{3 \times 3} \\
\mathbf{0}_{3 \times 3} & \mathbf{J}_{\omega}^{-1}
\end{array}\right)\left(\mathbf{s}-\mathbf{s}^{*}\right) \\
& =-\lambda\left(\begin{array}{c}
\mathcal{F} \mathbf{t}_{A}-\mathcal{F}_{A_{A^{*}}} \\
{ }_{\mathcal{F}} \mathbf{R}_{A} \theta \mathbf{u}
\end{array}\right)
\end{aligned}
$$

we obtain after simple developments:

$$
\begin{aligned}
\boldsymbol{v} & =-\lambda\left(\left({ }^{\mathcal{F}} \mathbf{t}_{A^{*}}-{ }^{\mathcal{F}} \mathbf{t}_{A}\right)+\left[{ }^{\mathcal{F}} \mathbf{t}_{A}\right]_{\times} \theta \mathbf{u}\right) \\
\boldsymbol{\omega} & =-\lambda \theta \mathbf{u}
\end{aligned}
$$

where

$$
\begin{aligned}
\mathcal{F}^{\mathcal{F}} \mathbf{t}_{A} & =\left(t_{x}, t_{y}, t_{z}\right)^{\top} \\
{ }^{\mathcal{F}} \mathbf{t}_{A^{*}} & =\left(t_{x}^{*}, t_{y}^{*}, t_{z}^{*}\right)^{\top}
\end{aligned}
$$

Considering task $\# 1$, since only the $x, y$ and $\alpha$ DOF are considered, the control law is given by:

$$
\left(\begin{array}{c}
\boldsymbol{v}_{x} \\
\boldsymbol{v}_{y} \\
\boldsymbol{\omega}_{\alpha}
\end{array}\right)_{\mathcal{F}}=-\lambda_{1}\left(\begin{array}{c}
t_{x}-t_{x}^{*} \\
t_{y}-t_{y}^{*} \\
\mathcal{F} \mathbf{R}_{A} \theta \mathbf{u}_{\alpha}
\end{array}\right)
$$

Similarly, for the second and third tasks, since only the $z$ and $\varphi$ DOF are considered, the control law is given by:

$$
\left(\begin{array}{c}
\boldsymbol{v}_{z} \\
\boldsymbol{\omega}_{\varphi}
\end{array}\right)_{\mathcal{F}}=-\lambda_{2}\left(\begin{array}{c}
t_{z}-t_{z}^{*} \\
\mathcal{F}^{\mathcal{R}} \mathbf{R}_{A} \theta \mathbf{u}_{\varphi}
\end{array}\right)
$$

The system switches automatically between task\#1, task\#2 and task\#3 when the norm of the error $\|\mathbf{e}\|$ is below a predefined threshold. In the experiments, the following values are used: $\left\|\mathbf{e}_{t}\right\|<\delta_{1}(0.5 \mu \mathrm{m})$ and $\left\|\mathbf{e}_{r}\right\|<\delta_{2}\left(0.2 \times 10^{-2} \mathrm{rad}\right)$ for the translation DOF and the rotation DOF, respectively.

To improve the convergence rate we implemented an adaptive gain (the gain increases when the error decreases):

$$
\lambda_{a d p}=\left(\lambda_{\max }-\lambda_{\min }\right) e^{-\kappa\|\mathbf{e}\|}+\lambda_{\text {min }},
$$

where $\lambda_{\max }$ and $\lambda_{\min }$ are the maximum and minimum values of $\lambda_{\mathrm{a}}$. The experimental results presented in this paper are obtained with $\lambda_{\min }=0.1$ and $\lambda_{\max }=1$. The parameter $\kappa$ allows the tuning of the exponential decreasing rate of the error $\mathbf{e}$ and $\|\mathbf{e}\|$ is its related norm. 


\section{$5 \quad$ Experimental results}

This section shows that the task of inserting two microparts $(400 \mu \mathrm{m} \times 400 \mu \mathrm{m} \times 100 \mu \mathrm{m})$ by their respective notches $(97 \mu \mathrm{m} \times 97 \mu \mathrm{m} \times 100 \mu \mathrm{m})$ with an assembly clearance ranging $1 \mu \mathrm{m}$ to $5 \mu \mathrm{m}$ is successfully achieved.

In a first time, the CAD model based tracking is evaluated on macroscale parts (Fig. 8). Its ability to work on the microscale is demonstrated when the depth-of-field of the imaging system is high enough to enable complete sharp views of the microparts. The tests show the efficiency of the tracking approach. In a second time, a full 3D visual control has been considered. Figure 9 shows some snapshots taken during the insertion (the CAD model is reprojected onto the micropart) and figure 10 shows some SEM (scanning electron microscope) images of the final assembly. The high quality of the tracking result in terms of precision and robustness expresses the relevance of the approach.

\subsection{Precision and robustness}

Figure 11 shows the evolution of the error $e_{x}, e_{y}$ and $e_{\alpha}$ versus the number of iterations $i$. The final positioning error along $x$ and $y$ axis of the micropart $\mathbf{A}(\operatorname{task} \# 1)$ are $e_{x}=3.52$ $\mu \mathrm{m}$ and $e_{y}=0.29 \mu \mathrm{m}$, respectively. The final orientation error in the same task is $e_{\alpha}=$ $0.30 \times 10^{-2} \mathrm{rad}$. Figure 12 shows the evolution of the errors $e_{z 1}$ and $e_{\varphi}$ versus the number of iterations $i$. The final positioning error along $z$ axis of the micropart $\mathbf{B}$ is $e_{z 1}=2.28 \mu \mathrm{m}$ and the final orientation error $e_{\varphi}$ with respect to the vertical axis, in the same task, is $e_{\varphi}$ $=0.13 \times 10^{-2} \mathrm{rad}$. These values are estimated from the encoders of the various angular and linear motions and the kinematic model.

The robustness of the approach presented in this paper can be underlined on several points. Firstly, multiple temporary and partial occlusions (by the gripper or by other microparts) of the microparts happened during the manipulation. That do not prevent the algorithm to track the parts. Secondly, it is not possible due to the small depth of field of the optical microscope, to get sharp images of the microparts. The tracking method based on the virtual visual servoing combined with a technique of robust M-estimation introduced directly in the control law allows to precisely track the microparts even when the tracked is partially blurred (see Fig. 13 and Extension 5 of this paper).

Figure 14 represents the microparts $\mathbf{A}$ and $\mathbf{B}$ trajectories during the achievement of the different assembly tasks as shown in Fig. 2. It can be noted that the previously proposed 3D control law achieves, as expected, a straight line trajectories of the microparts. This is especially visible for the displacement of the micropart $\mathbf{A}$ located on the 3 DOF positioning platform.

\subsection{Assembly cycle time}

The assembly of two microparts (i.e. A and B) comprises three tasks $[$ task\#1, task\#2 and task\#3] as previously presented: planar positioning of $\mathbf{A}$, spatial positioning of $\mathbf{B}$ and 

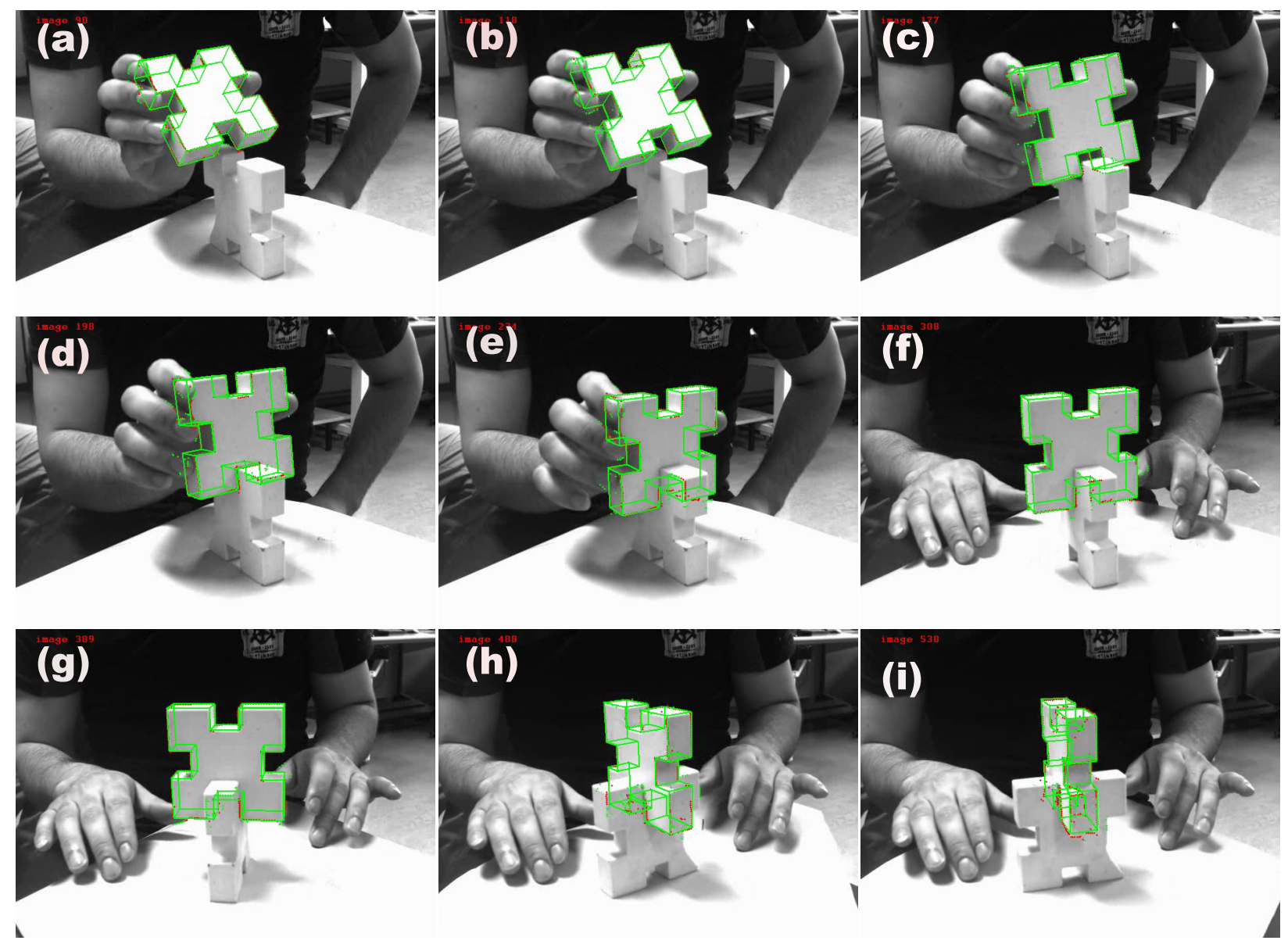

Figure 8: CAD model based tracking algorithm applied to a $80 \mathrm{~mm} \times 80 \mathrm{~mm} \times 20 \mathrm{~mm}$ macropart: (a) shown the tracker initialization. (b) and (c) intermediate positions of the tracked part. (d) illustrates the beginning of the insertion of the tracked part into the second part. (e) shown the end of the manual assembly process. (f) to (i) represent a rotation of $\pi / 4$ of the performed assembly which shows the tracker efficiency by changing the perspective of the part. This experiment is illustrated by the Extensions 1 (refer to the Appendix A).

insertion of $\mathbf{B}$ into $\mathbf{A}$, respectively. To compute the required time to built a 3D microstructure, we have considered the positioning time (time required to position the both microparts at their respective insertion positions) and the insertion time (required time to insert the micropart $\mathbf{B}$ into $\mathbf{A}$ ).

Let $T_{\text {positioning }}$ which corresponds to the time required to perform task\#1 (positioning time of the micropart $\mathbf{A}$ ) and $T_{\text {insertion }}$ be the positioning and insertion time required to achieve the task\#2 and task\#3.

Table 3 summarizes the values obtained when the assembly is performed ten times. Despite the caution taken to perform the insertion, the latter takes less time than the position- 

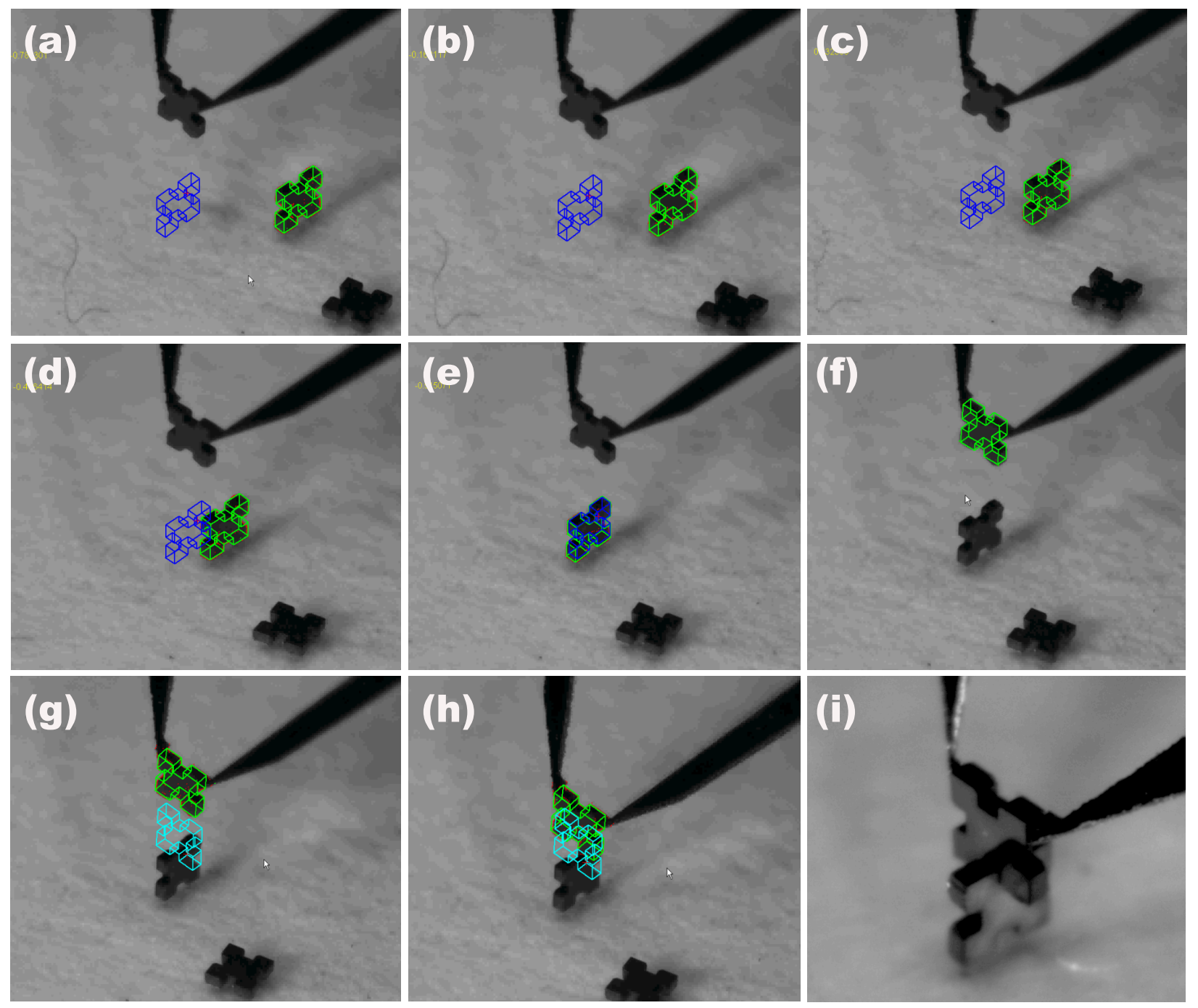

Figure 9: Successful assembly of the microscale parts A and B. (a) the tracker initialization. (b), (c) and (d) intermediate positions of the micropart A. (e) end of the positioning of A. (f) beginning of the tracking of the micropart B. (g) and (h) intermediate positions of the micropart B. (i) zoom of the final 3D microstructure. These results are shown in the Extension 2 of this paper.

ing, about the third of the positioning time. The mean values of $T_{\text {positioning }}$ and $T_{\text {insertion }}$ are 28.8 seconds (with a standard deviation of 3.5 seconds) and 12.0 seconds (with a standard deviation of 0.6 seconds), respectively. Then the mean cycle time for the assembly is 40.8 seconds (with a standard deviation of 3 seconds) which is low enough to foresee industrial use of the concepts. To our knowledge, this quality of result has never been presented in the literature before. This based on the obtained precision with a short assembly cycle times. 


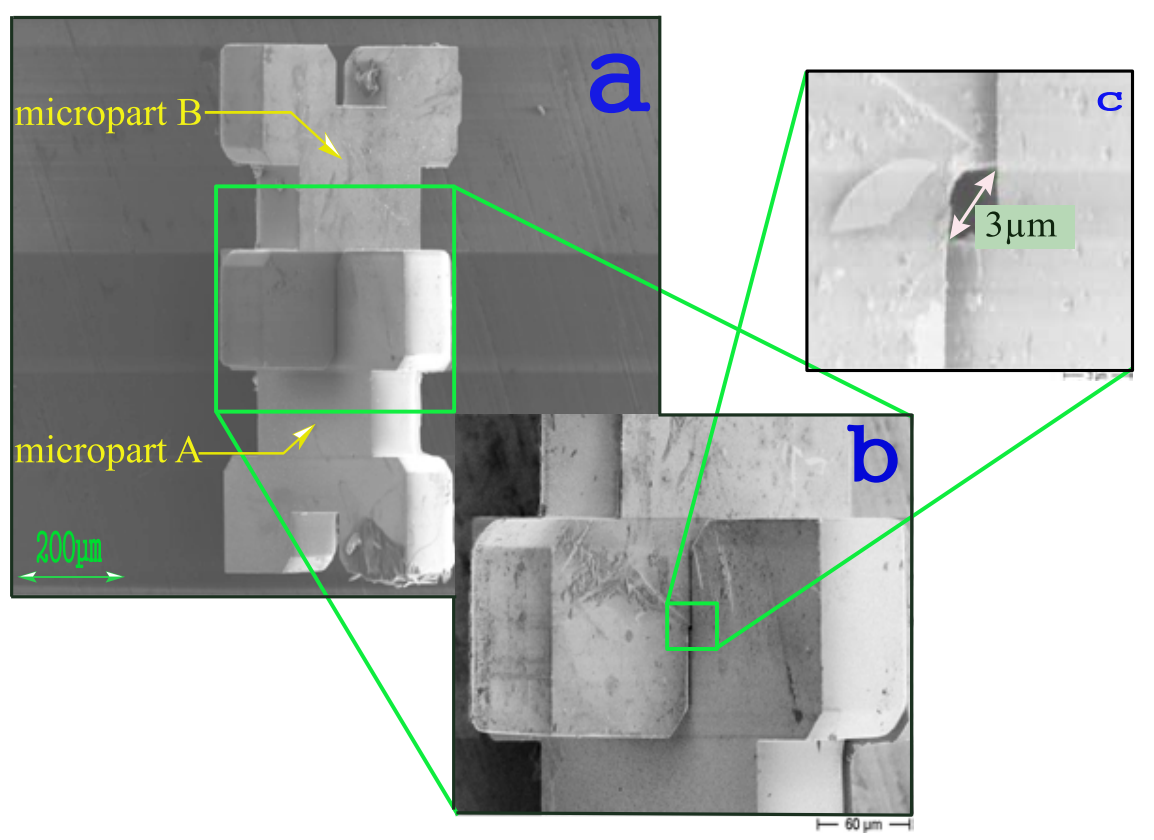

Figure 10: Some SEM views of the 3D structure after assembly. The small size of the assembly clearance is visible in the zoomed image (c). This experiment is illustrated in Extension 4.

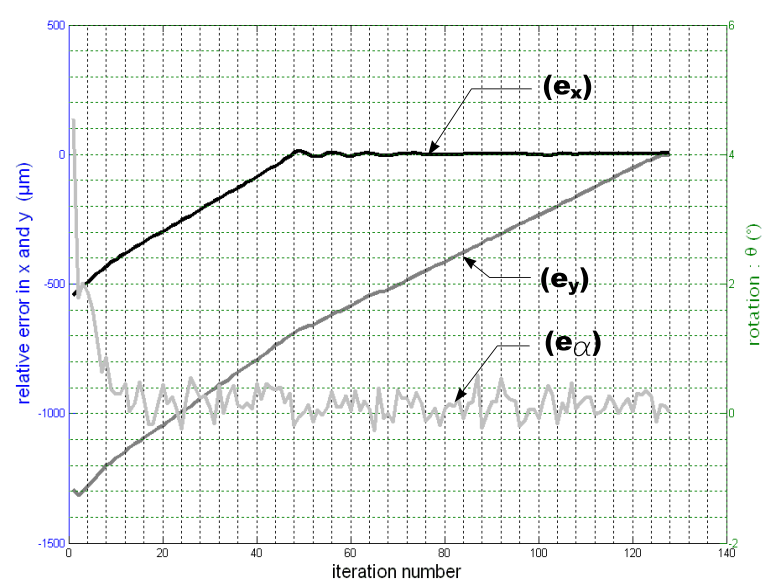

Figure 11: Errors on the positioning along $x, y$ and the orientation $\alpha$ errors versus the number of iterations $i$.

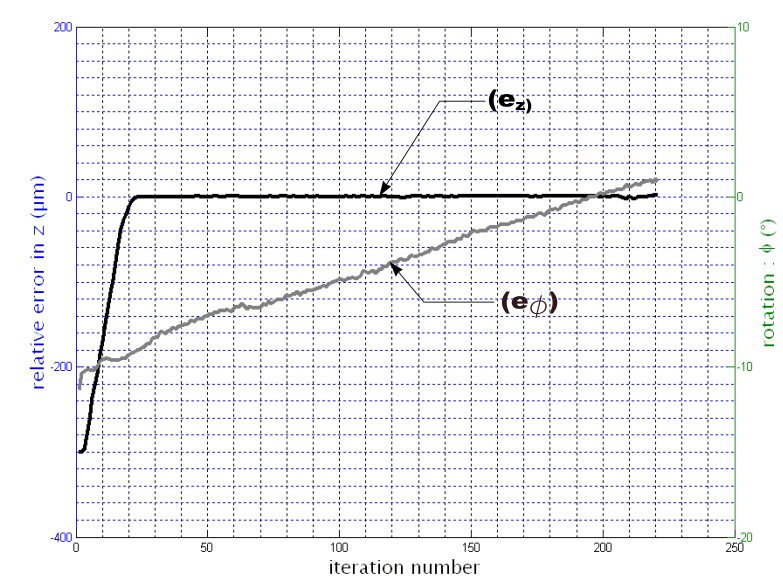

Figure 12: Errors on the positioning along $z$ and the orientation $\varphi$ versus the number of iterations $i$.

\subsection{Complex MEMS structures assembly}

The techniques presented previously are also validated on a more complex microassembly process. It consists in a fully-automatic assembly of five simple MEMS (the same kind of 


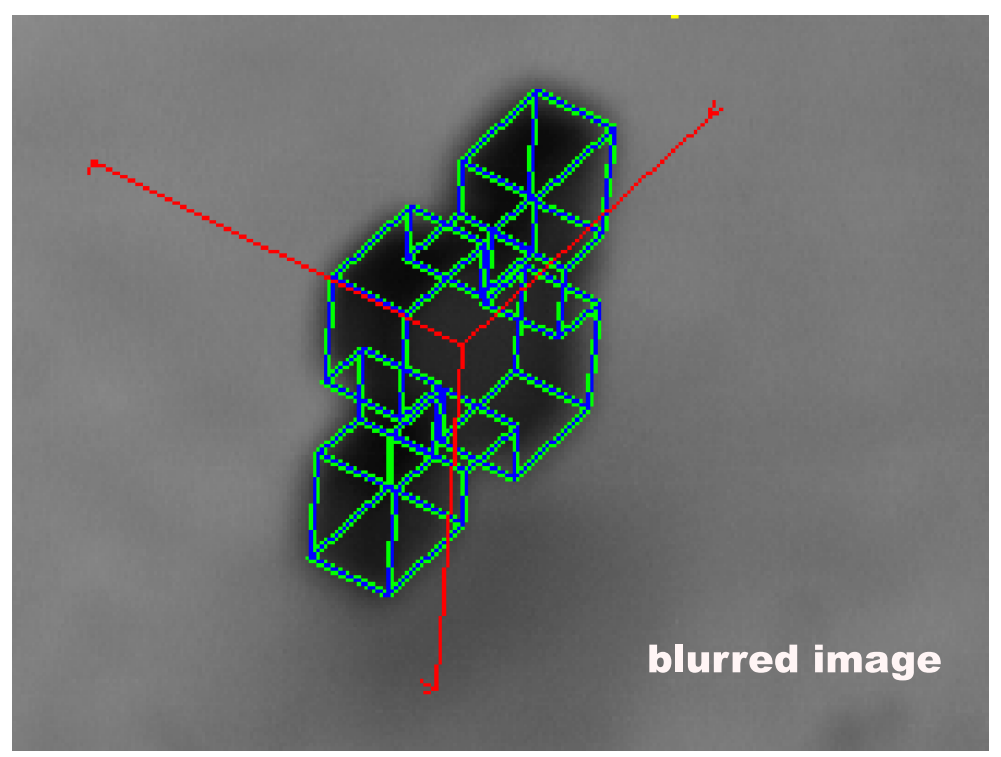

Figure 13: Illustration of the robustness of the tracking algorithm in the case of partial blurred images.

micropart used for the first validation) to build 3D MEMS on 3 levels as illustrated by Fig. 15 . The microparts are previously randomly positioned (with different orientations) within a radius of $1.5 \mathrm{~mm}$ around the center of the positioning platform with various orientations ranging between 0 to $\pi / 4$. This has been studied in the context of recent works (Tamadazte et al., 2009) using a control law based on a multiple scale image-based visual servoing.

Let $\mathcal{R}_{A}, \mathcal{R}_{B}, \mathcal{R}_{C}, \mathcal{R}_{D}$ and $\mathcal{R}_{E}$ be the frames linked to the current positions of each MEMS sample (i.e. A, B, C, D and E) to assemble and $\mathcal{R}_{A^{*}}, \mathcal{R}_{B^{*}}, \mathcal{R}_{C^{*}}, \mathcal{R}_{D^{*}}$ and $\mathcal{R}_{E^{*}}$ their respective desired positions (see, Fig. 16). The homogeneous transformations between the camera frame and the current positions of each MEMS sample are noted ${ }^{c} \mathbf{M}_{A},{ }^{c} \mathbf{M}_{B},{ }^{c} \mathbf{M}_{C}$, ${ }^{c} \mathbf{M}_{D}$ and ${ }^{c} \mathbf{M}_{E} \cdot{ }^{c} \mathbf{M}_{A^{*}},{ }^{c} \mathbf{M}_{B^{*}},{ }^{c} \mathbf{M}_{C^{*}},{ }^{c} \mathbf{M}_{D^{*}}$ and ${ }^{c} \mathbf{M}_{E^{*}}$ represent the homogeneous transformations between the camera frame $\mathcal{R}_{c}$ and the desired positions of these MEMS samples.

The switch between the different positioning tasks (control of the positioning platform) and insertion (control of the micromanipulator) for successive microparts is done when the threshold of translation and rotation errors is reached $\left(\left\|\mathbf{e}_{t}\right\|<\delta_{1}(0.5 \mu \mathrm{m})\right.$ and $\left\|\mathbf{e}_{r}\right\|<\delta_{2}$ $\left.\left(0.2 \times 10^{-2} \mathrm{rad}\right)\right)$. The same threshold is applied for all the microassembly process tasks.

The desired position $\left({ }^{\mathcal{F}} \mathbf{M}_{A^{*}}\right)$ of the first micropart $\mathbf{A}$ is defined manually (initialization step). When $\mathbf{A}$ is perfectly positioned ( $\left\|\mathbf{e}_{t}\right\|<\delta_{1}$ and $\left\|\mathbf{e}_{r}\right\|<\delta_{2}$ ), the final position of the second micropart $\mathbf{B}$ is computed automatically by:

$$
{ }^{\mathcal{F}} \mathbf{M}_{i}={ }^{\mathcal{F}} \mathbf{M}_{A}{ }^{A} \mathbf{M}_{i}
$$

with $i$ the micropart label $(\mathbf{A}, \mathbf{B}, \mathbf{C}, \mathbf{D}$ or $\mathbf{E})$ and ${ }^{A} \mathbf{M}_{i}$ represents the transformation matrix between the frame linked to the micropart $\mathbf{A}$ to the frame linked to the micropart $i$. For 


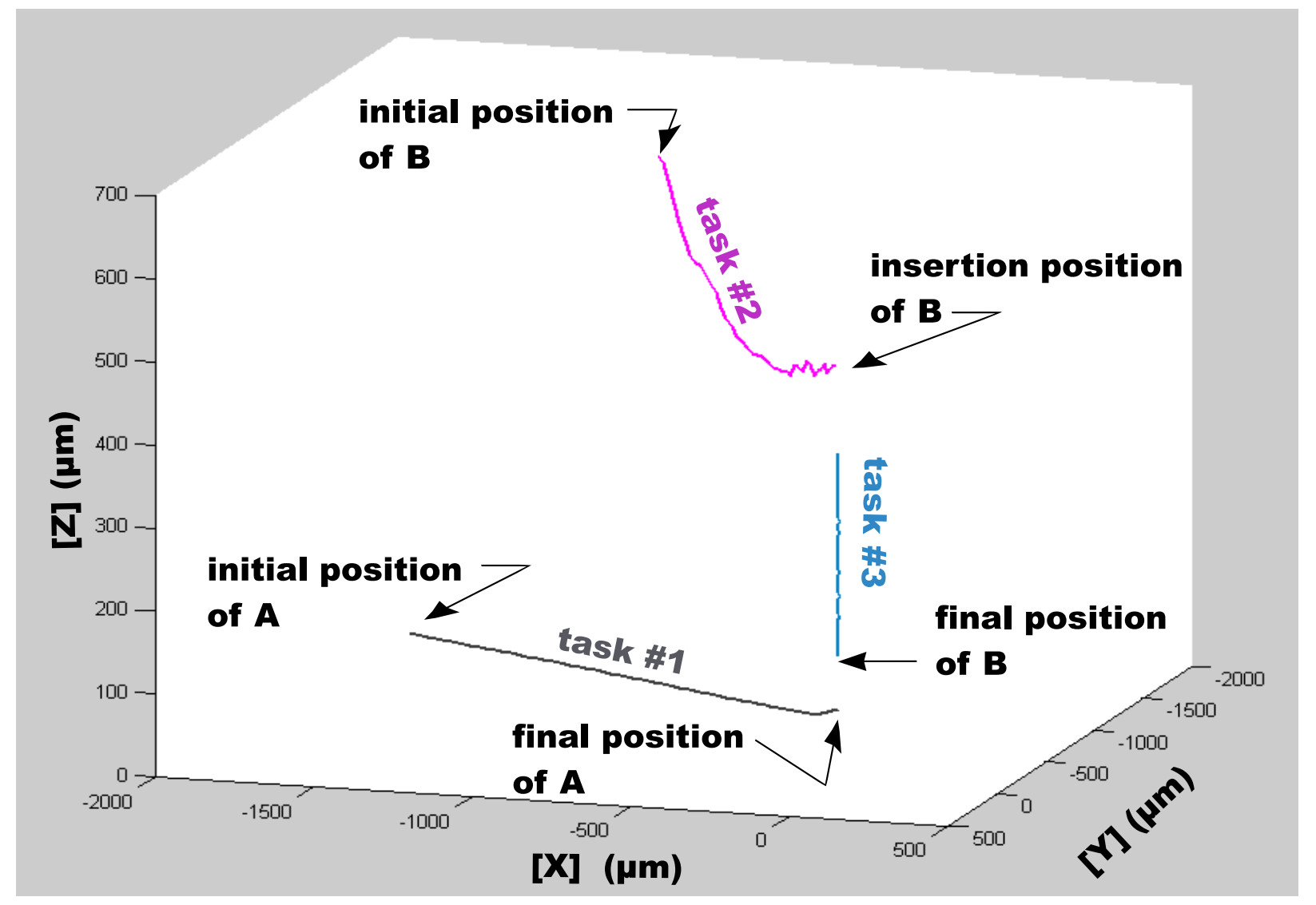

Figure 14: Illustration of the different trajectories obtained with the pose-based visual control.

instance, the transformation matrix (vertical translation) between the desired pose of the micropart $\mathbf{A}$ and the desired pose of the micropart $\mathbf{B}$ (see Fig. 17) is given by:

$$
{ }^{A} \mathbf{M}_{B}=\left(\begin{array}{cccc}
1 & 0 & 0 & 0 \\
0 & 1 & 0 & 0 \\
0 & 0 & 1 & 300 \\
0 & 0 & 0 & 1
\end{array}\right)(\mu m)
$$

Figure 18 illustrates a sequence of images from the optical videomicroscope system showing the process of the automatic microassembly of five silicon microparts of size of $400 \times$ $400 \times 100 \mu \mathrm{m}^{3}$. The Fig. 18.(a) illustrates the first step which concerns the tracking and the positioning of micropart A. Figure 18.(b) represents the tracking and placing of micropart B. Figure 18.(c) shows the tracking of micropart A and the positioning of both microparts $[\mathbf{A}+\mathbf{B}]$. Figure 18.(d) shows the tracking and insertion of micropart $\mathbf{C}$ into sub-assembly $[\mathbf{A}+\mathbf{B}]$. Figure 18.(e) represents the tracking of micropart $\mathbf{A}$ and positioning of the subassembly $[\mathbf{A}+\mathbf{B}+\mathbf{C}]$. Figure 18.(f) illustrates the tracking and the insertion of micropart 
Table 3: Some assembly cycle times. The microparts are randomly positioned with different orientations ranged between 0 to $\pi / 4$ within a radius of $1.5 \mathrm{~mm}$ around the center of the positioning platform.

\begin{tabular}{c|c|c|c}
\hline $\mathrm{N}$ & $T_{\text {positioning }}(\mathrm{sec})$ & $T_{\text {insertion }}(\mathrm{sec})$ & $T_{\text {complete }}(\mathrm{sec})$ \\
\hline 1 & 25.9 & 13.9 & 39.8 \\
2 & 29.7 & 11.7 & 41.4 \\
3 & 22.0 & 12.0 & 34.0 \\
4 & 28.3 & 11.5 & 39.8 \\
5 & 35.7 & 11.7 & 47.4 \\
6 & 31.3 & 12.0 & 43.3 \\
7 & 28.8 & 11.8 & 40.6 \\
8 & 29.4 & 11.9 & 41.3 \\
9 & 27.8 & 11.9 & 39.7 \\
10 & 29.4 & 11.7 & 41.1 \\
\hline Mean & $\mathbf{2 8 . 8}$ & $\mathbf{1 2 . 0}$ & $\mathbf{4 0 . 8}$ \\
\hline Standard deviation & $\mathbf{3 . 5}$ & $\mathbf{0 . 6}$ & $\mathbf{3 . 0}$ \\
\hline
\end{tabular}

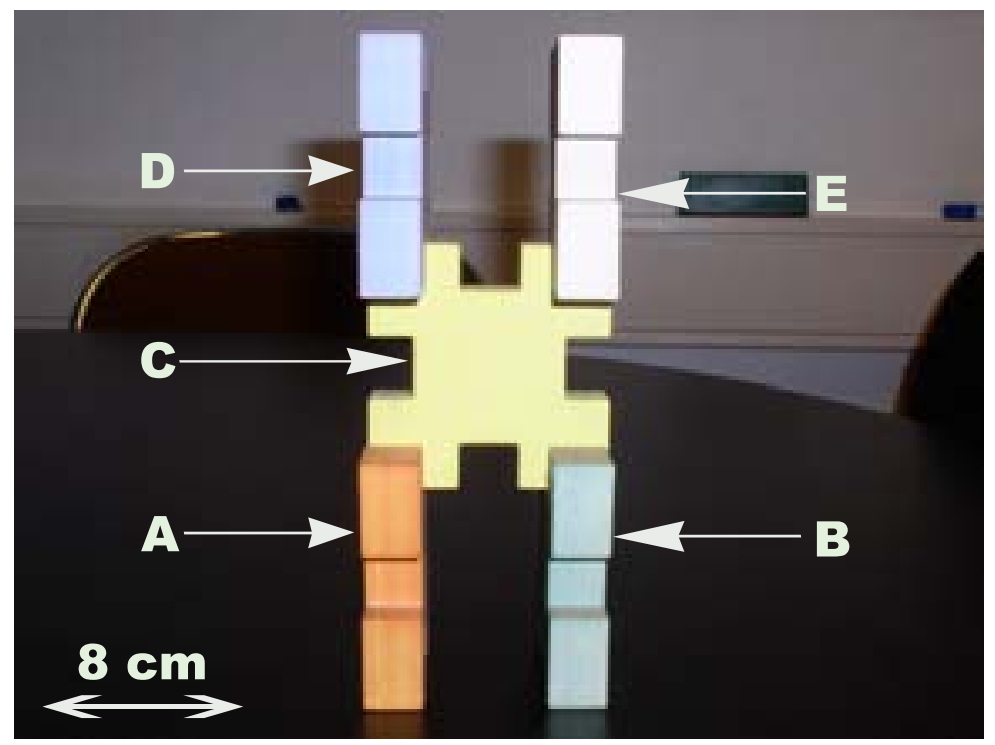

Figure 15: Illustration of the assembly to achieve with macroscale parts. 


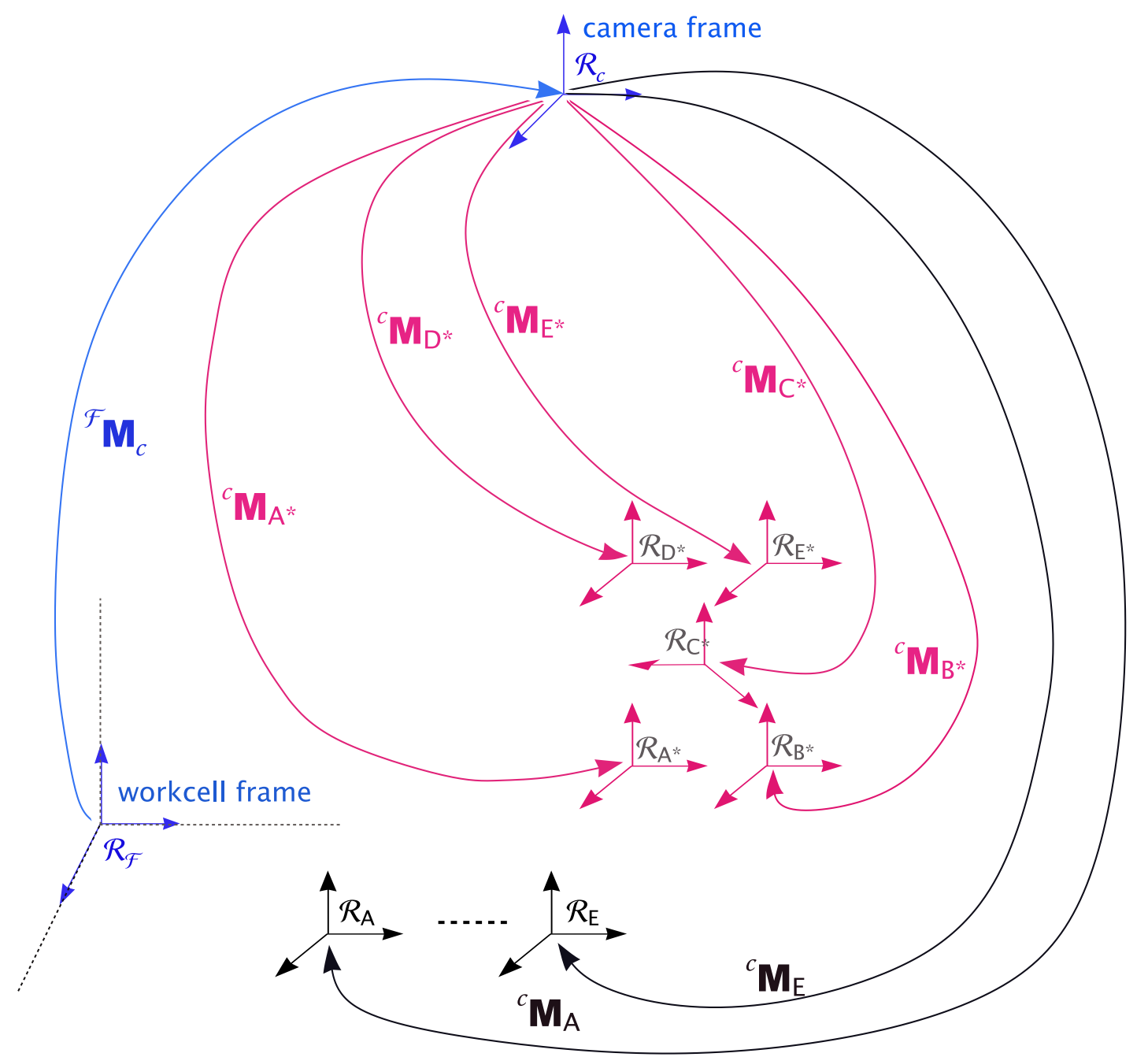

Figure 16: Illustration of the frames used in the experiments.

D into the sub-assembly $[\mathbf{A}+\mathbf{B}+\mathbf{C}]$. Figure 18.(g) illustrates a zoom on the end of the insertion of the micropart $\mathbf{C}$ into the sub-assembly $[\mathbf{A}+\mathbf{B}]$. Figure 18.(h) shows the end of the assembly process which concerns the insertion of the fifth micropart $\mathbf{E}$ into sub-assembly $[\mathbf{A}+\mathbf{B}+\mathbf{C}+\mathbf{D}]$. Figure 18.(i) represents a zoom on the final 3D microassembly. This experiment is presented in the Extension 3 of this paper (refer to the Appendix A for details).

It can be noted that the MEMS obtained (see, Fig. 18.(i)) using the proposed approaches is more precise than the MEMS assembled by a human operator (see, Fig. 19). The latter has been assembled in a tele-operated mode using a joystick by a human operator who has about one year of experience in the use of the microassembly workcell.

It is also possible to extend the work presented in this paper to the automatic microma- 


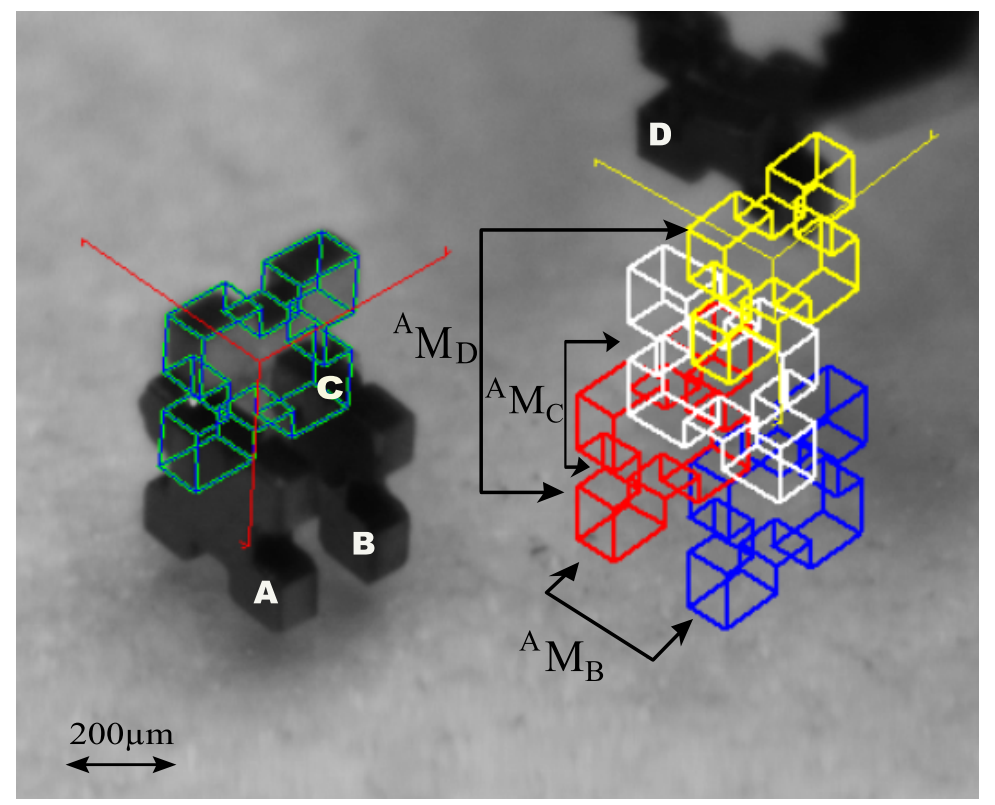

Figure 17: Illustration of an intermediate step of the microassembly process. Positioning task of the micropart $\mathbf{C}$ under the gripper system followed by the insertion task of micropart $\mathbf{D}$ into sub-assembly $[\mathbf{A}+\mathbf{B}+\mathbf{C}]$.

nipulation and microassembly of MEMS using SEM or TEM imaging systems. The challenge of the manipulation inside the SEM is to perform visual servoing tasks. Aiming of this, the tracker proposed is tested and validated on SEM images of a silicon micropart, as illustrated in Fig. 20. These first results promise an extension of the work proposed in this paper to the nanoscale domain.

\section{Conclusion and future perspectives}

\subsection{Conclusion}

The problem of robotic microassembly of 3D MEMS devices has been studied through this paper. Despite the wealth and important scientific contributions in this field, there is still much efforts to bring to get the famous flexible, modular, versatile, repeatable and precise microassembly process. Almost all the works proposed in the literature so far concerns the handling (positioning, orientation, gripping, picking, placing, etc.) and microassembly, that mainly relates to the peg-in-a-hole kind of insertion. As a complement, this paper was more focused on performing relatively complex assemblies. The solution proposed to this problem was the development of a vision feedback strategy, namely 3D visual control. Therefore, this paper addressed the automation of assembly from conceptual viewpoint to a practical solution compatible with industrial constraints. These may be multiple as the need to have flexible, versatile, reconfigurable micro-assembly workcells which can be work on small and 

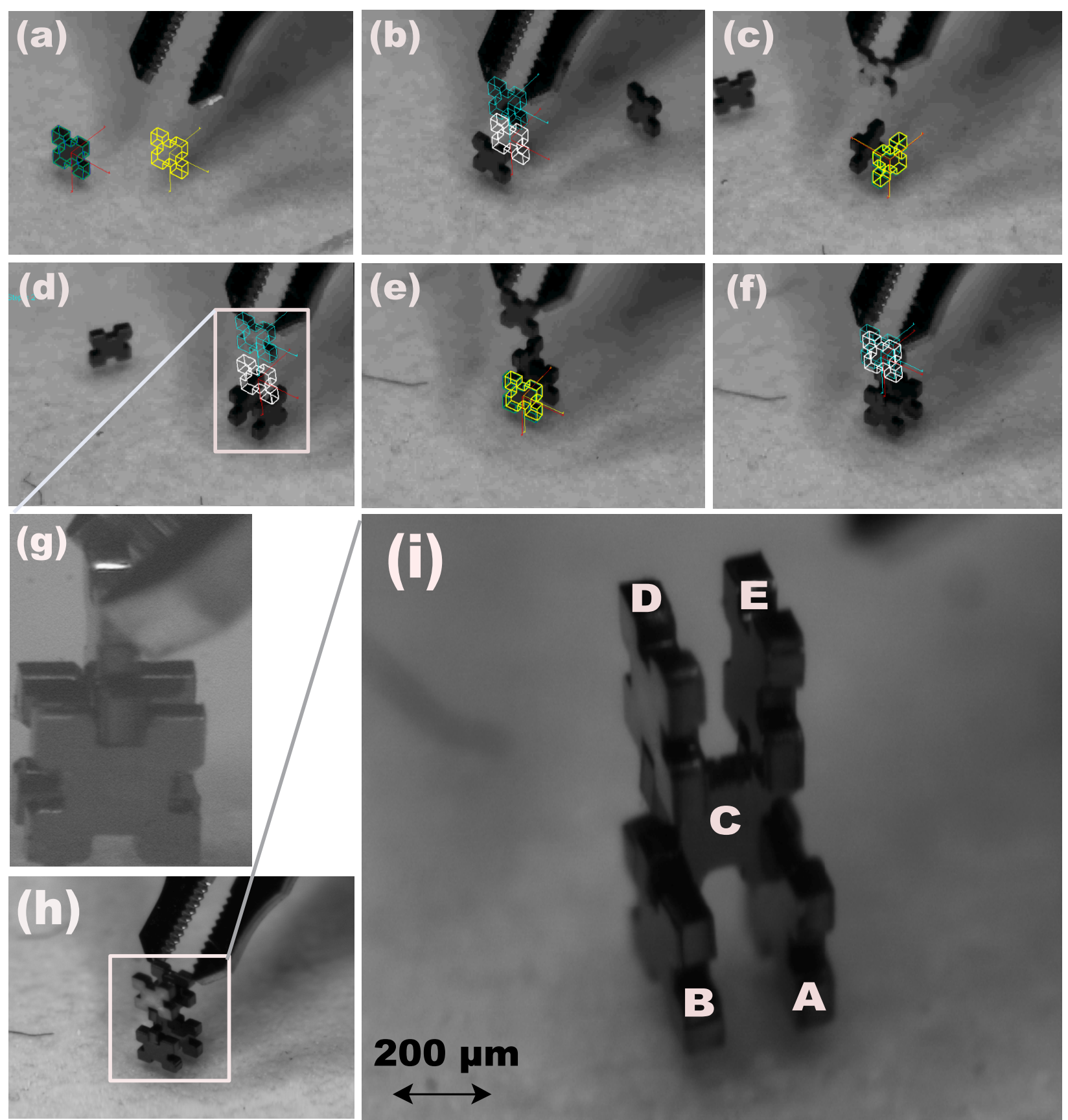

Figure 18: Sequence of images captured during the microassembly process. 


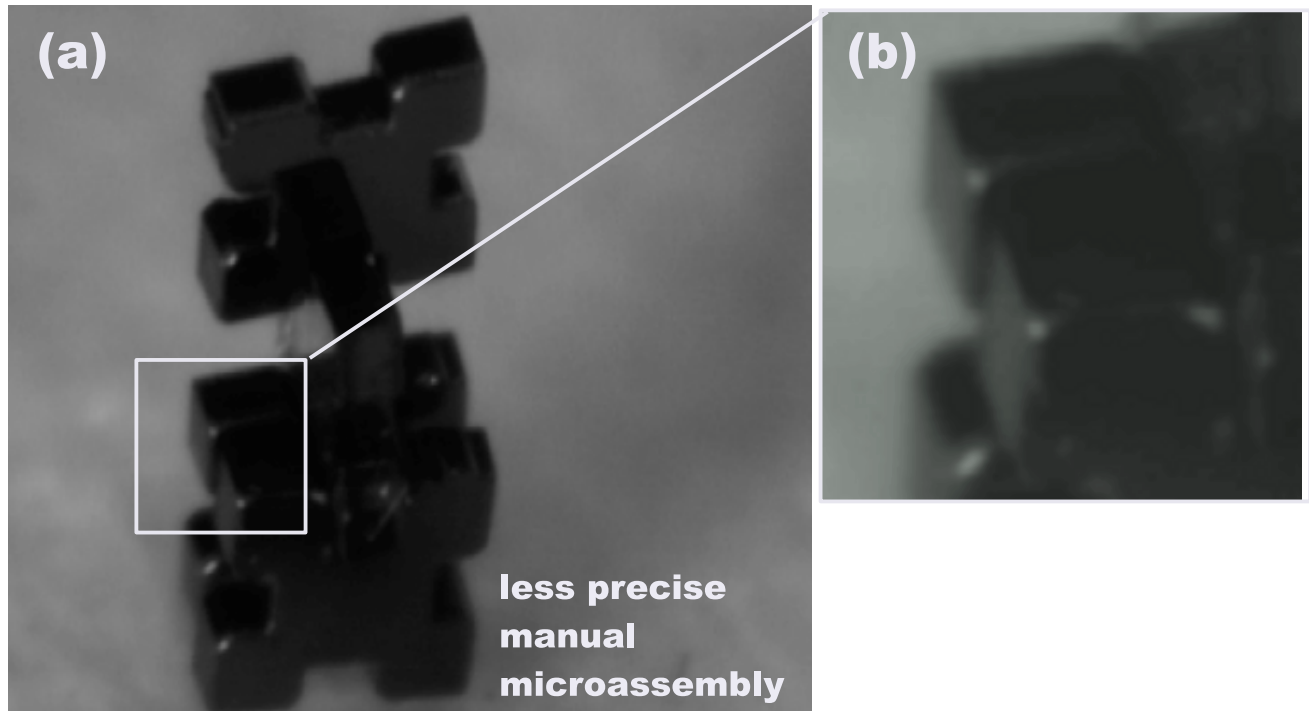

Figure 19: MEMS assembled in tele-operated mode.
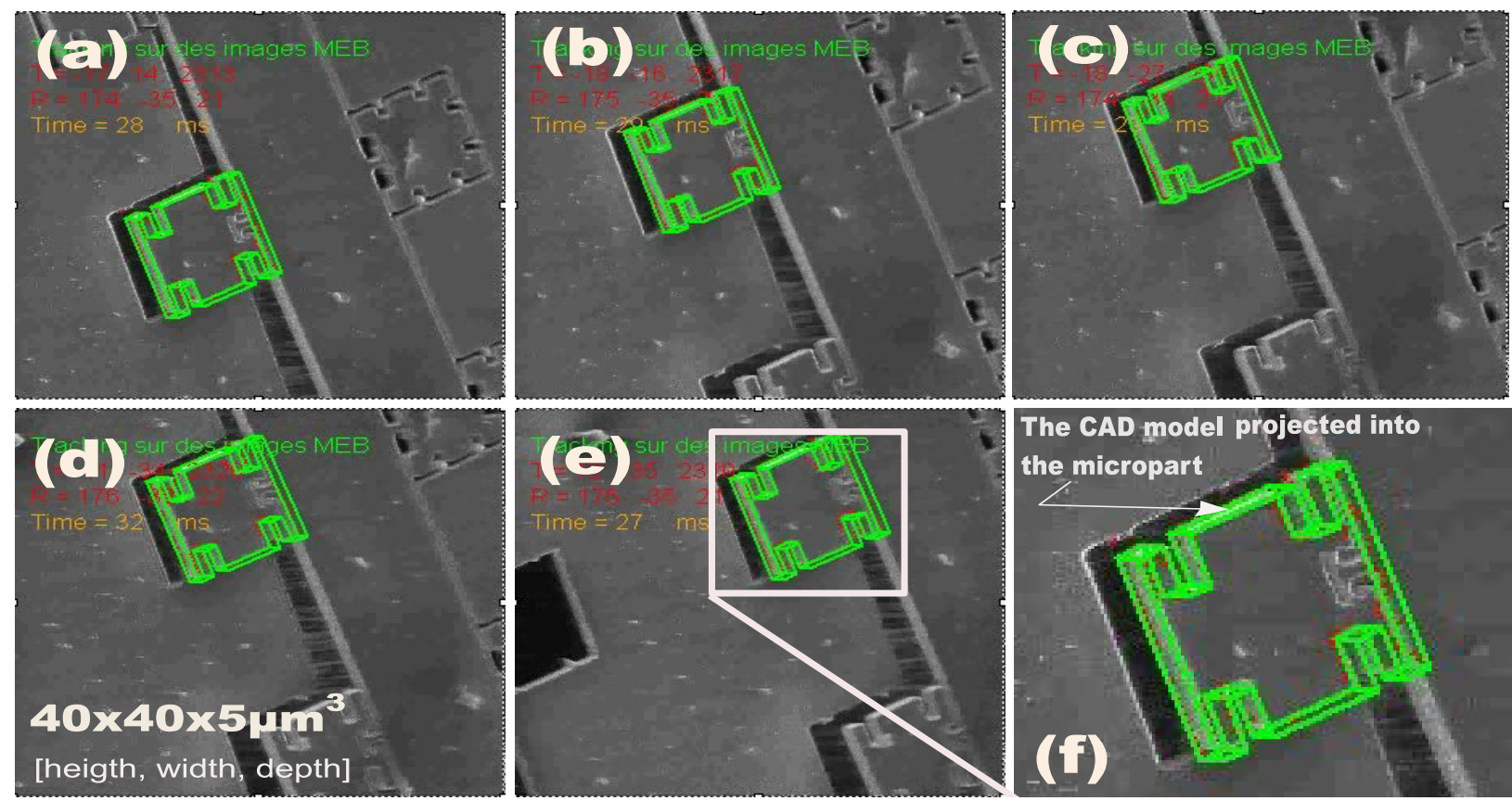

Figure 20: MEMS sequence with CAD model projected on the micropart. A pallet of silicon micropart of $40 \mu \mathrm{m} \times 40 \mu \mathrm{m} \times 5 \mu \mathrm{m}$ is placed on the SEM. In this example only one object is tracked. However, we could consider the tracking of two or more microparts at the same time. Figures (a) to (e) show the application of the tracker on a SEM sequence of images and the figure (f) illustrates a zoom on a micropart tracked. 
medium series using different shape and size sample MEMS. This with a good repeatability, precision and lows cycle time.

The experiments show the validity of the proposed method. Firstly, an automatic microassembly was performed. It consists on fitting together two $400 \mu \mathrm{m} \times 400 \mu \mathrm{m} \times 100$ $\mu \mathrm{m}$ microparts by their $97 \mu \mathrm{m} \times 97 \mu \mathrm{m} \times 100 \mu \mathrm{m}$ notches. Secondly, a more complex microassembly was performed that consists of a fully-automatic microassembly of five simple microstructures to built a 3D MEMS on three levels. The experimental setup mainly comprises a five DOF robotic structure ( $x y \alpha$ positioning platform and $z \varphi$ micromanipulator), a four DOF microhandling system (two DOF by finger), an imaging system (videomicroscope) and a controlled environment.

3D features (poses of the both microparts) were delivered by a real-time visual tracking algorithm. This algorithm consists of the association between the 2D features extracted from images and their corresponding features in the CAD model. Despite the partial or temporary occlusions by the gripper during the positioning tasks, the algorithm remains robust and very precise. The tracker thus delivers highly precise 3D measures which were used to implement a precise 3D pose-based visual control. The tasks were performed with the following precision: the positioning error reaches $0.3 \mu \mathrm{m}$ (for $x y z$ stages) and $0.35 \times 10^{-2}$ rad for the orientation stages $(\alpha \varphi)$.

The time required to achieve an assembly was also in the focus attention. The mean time spent to perform the microassembly was computed for various assemblies. A mean time of about 28.8 seconds is necessary to accomplish the positioning of both microparts to their respective insertion positions and 12.0 seconds for their insertion. So the mean of the assembly cycle time is less than 41 seconds. This makes the proposed automated platform 15 times faster and more precise than a human operator and an open way for industrial applications, more precisely to assemble hybrid MEMS in small and medium series.

\subsection{Future perspectives}

Future work will concern the improvement of the efficiency of the proposed concepts. Namely, exhaustive experimentations will be done and a statistical study will be made about the success rate, causes of the failures (control errors, tracking errors, occlusions, adhesion forces, electrostatic effects, etc). Through such studies, lessons will be learned and solutions will be proposed to improve the efficiency of this process.

On the other hand, our future works will obviously move towards:

- increasing the number of components to assemble;

- increasing the complexity of the operations (Fig. 21);

- increasing the production volume and reducing the assembly cycle time;

- reducing the microparts size to a few ten micrometers. 

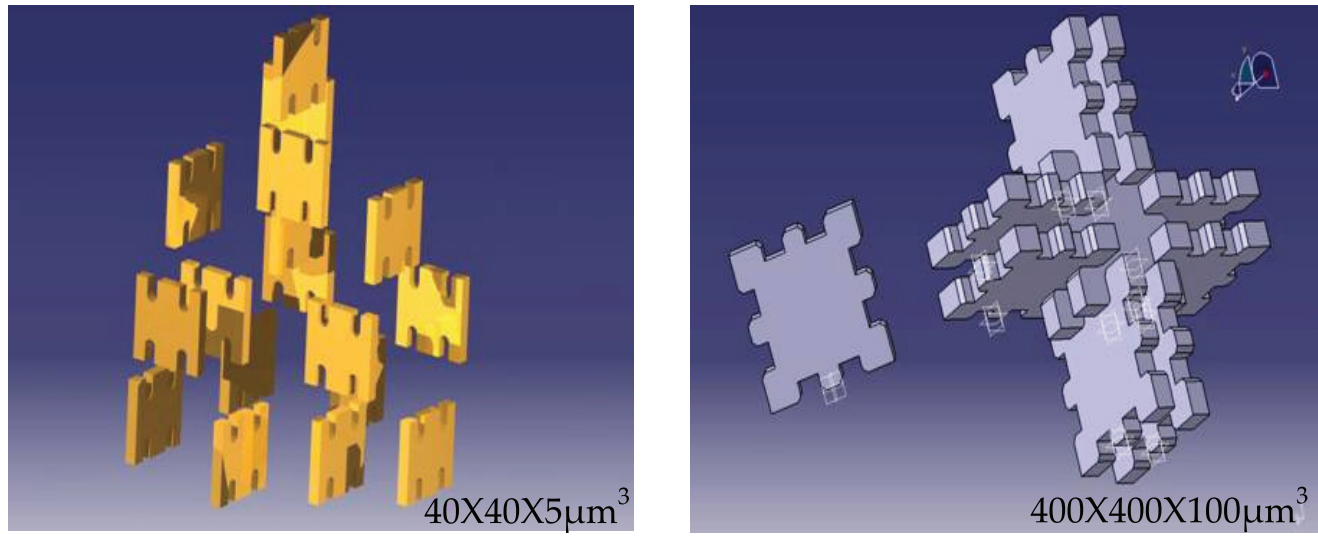

Figure 21: Some examples of 3D complex assembly.

\section{Aknowledgements}

This work is partially conducted with financial support from the project "Hybrid Ultra Precision Manufacturing Process Based on Positional and Self assembly for Complex MicroProducts (HYDROMEL NMP2-CT-2006-026622)" funded by the European Commission.

The authors thank Miss Joanna Farrow, English teacher at ENSMM (National Engineering School of Mechanics and Microtechnics) of Besançon for these multiple proof-reading of this paper.

\section{A Transformation definition}

In this paper, we defined a rigid transformation between a frame $\mathcal{R}_{a}$ and a frame $\mathcal{R}_{b}$ by an homogeneous matrix ${ }^{a} \mathbf{M}_{b}$ defined as in (Murray et al., 1994) by:

$$
{ }^{a} \mathbf{M}_{b}=\left[\begin{array}{cc}
{ }^{a} \mathbf{R}_{b} & { }^{a} \mathbf{t}_{b} \\
0 & 1
\end{array}\right]
$$

where ${ }^{a} \mathbf{R}_{b}$ is the rotation matrix between the frame $\mathcal{R}_{a}$ and the frame $\mathcal{R}_{b}$ and ${ }^{a} \mathbf{t}_{b}$ the translation vector between the frame $\mathcal{R}_{a}$ and the frame $\mathcal{R}_{b}$. It is also possible to note the pose by the vector:

$$
{ }^{a} \mathbf{r}_{b}=\left({ }^{a} \mathbf{t}_{b}, \theta \mathbf{u}\right)
$$

with $\theta \mathbf{u}$ representing a rotation of an angle $\theta$ carried by the an axis $\mathbf{u}$ and given by: 


$$
\theta \mathbf{u}=\frac{1}{2 \operatorname{sinc} \theta}\left(\begin{array}{l}
r_{32}-r_{23} \\
r_{13}-r_{31} \\
r_{21}-r_{12}
\end{array}\right)
$$

with

$$
\theta=\cos ^{-1}\left(1 / 2\left(r_{11}+r_{22}+r_{33}-1\right)\right)
$$

where $\operatorname{sinc} \theta=\sin \theta / \theta$ and $r_{11}, r_{12}, \ldots, r_{33}$ are the terms of the rotation matrix ${ }^{a} \mathbf{R}_{b}$ as:

$$
\mathbf{R}=\left(\begin{array}{lll}
r_{11} & r_{12} & r_{13} \\
r_{21} & r_{22} & r_{23} \\
r_{31} & r_{32} & r_{33}
\end{array}\right)
$$

\section{B Notations}

\begin{tabular}{c|l}
\hline Notations & Description \\
\hline $\mathbf{S}$ & current pose \\
$\mathbf{s}^{*}$ & desired pose \\
$\mathbf{e}$ & error between $\mathbf{s}$ and $\mathbf{s}^{*}$ \\
$\dot{\mathbf{s}}$ & time variations of $\mathbf{s}$ \\
$\mathbf{v}$ & velocity screw of the virtual camera \\
${ }^{a} \mathbf{M}_{b}$ & rigid transformation matrix between the frames $\mathcal{R}_{a}$ and $\mathcal{R}_{b}$, respectively \\
${ }^{a} \mathbf{r}_{b}$ & vector pose between the frames $\mathcal{R}_{a}$ and $\mathcal{R}_{b}$, respectively \\
${ }^{a} \mathbf{R}_{b}$ & rotation matrix between the frames $\mathcal{R}_{a}$ and $\mathcal{R}_{b}$, respectively \\
${ }^{a} \mathbf{t}_{b}$ & translation vector between the frames $\mathcal{R}_{a}$ and $\mathcal{R}_{b}$, respectively \\
$\theta \mathbf{u}$ & rotation of an angle $\theta$ carried by the axis $\mathbf{u}$ \\
$\mathcal{R}_{\mathcal{F}}$ & frame linked to the workcell base \\
${ }^{c} \mathbf{M}_{a}$ & 3D pose of a expressed in the camera frame $\mathcal{R}_{c}$ \\
${ }^{a} \mathbf{M}_{a^{*}}$ & 3D error to regulate to zero between current pose of $a$ and desired pose of $a^{*}$ \\
$\mathbf{L}_{\omega}$ & 3D interaction matrix \\
$\boldsymbol{v}$ & translation velocity \\
$\boldsymbol{\omega}$ & rotation velocity \\
$\mathbf{D}$ & diagonal weighting matrix \\
$\psi$ & robust function \\
$d_{\perp}(\mathbf{p}, \mathbf{l}(\mathbf{r}))$ & orthogonal distance between $\mathbf{p}$ and the line $\mathbf{l}(\mathbf{r})$ \\
\hline
\end{tabular}




\section{$\mathrm{C}$ Index to multimedia extensions}

\begin{tabular}{c|c|l}
\hline Extension & Type & Description \\
\hline $\mathbf{1}$ & video & $\begin{array}{l}\text { validation of the CAD model based tracking algorithm using } \\
80 \mathrm{~mm} \times 80 \mathrm{~mm} \times 20 \mathrm{~mm} \text { size macro-objects and peg-in-hole } \\
\text { camera equipped with a conventional objective-lens }[4.54 \mathrm{MB}] \text { avi }\end{array}$ \\
\hline $\mathbf{2}$ & video & $\begin{array}{l}\text { automatic MEMS assembly of two } 400 \mu \mathrm{m} \times 400 \mu \mathrm{m} \times 100 \mu \mathrm{m} \\
\text { size silicon microparts using the proposed tracking and visual } \\
\text { servoing techniques }[6.54 \mathrm{MB}] \text { avi }\end{array}$ \\
\hline $\mathbf{3}$ & video & $\begin{array}{l}\text { automatic more complex MEMS assembly of five } \\
400 \mu \mathrm{m} \times 400 \mu \mathrm{m} \times 100 \mu \mathrm{m} \text { size silicon microparts }[4.93 \mathrm{MB}] \text { avi }\end{array}$ \\
\hline $\mathbf{4}$ & video & $\begin{array}{l}\text { validation of the CAD model based tracking algorithm using } \\
40 \mu \mathrm{m} \times 40 \mu \mathrm{m} \times 5 \mu \mathrm{m} \text { size silicon micro-objects placed inside } \\
\text { a SEM }[6.34 \mathrm{MB}] \text { avi }\end{array}$ \\
\hline $\mathbf{5}$ & video & $\begin{array}{l}\text { CAD model based tracking algorithm application to partially blurred } \\
\text { images }[2.61 \mathrm{MB}] \text { avi }\end{array}$ \\
\hline
\end{tabular}

\section{References}

J. J. Abbott, Z. Nagy, F. Beyeler, and B. J. Nelson. 2007. Robotics in the small, Part I: Microrobotics. IEEE Robotics and Automation Magazine, 14(2):92-103.

J. Agnus, P. Nectoux, and N. Chaillet. 2005. Overview of microgrippers and design of a micromanipulation station based on mmoc microgripper. In IEEE International Symposium on Computational Intelligence in Robotics and Automation, 117-123, Finland.

M. Ammi and A. Ferreira. 2007. Robotic assisted micromanipulation system using virtual fixtures and metaphors. IEEE International Conference Robotics and Automation, 454460.

M. Ammi, V. Fremont, and A. Ferreira. 2005. Flexible microscope calibration using virtual pattern for 3D telemicromanipulation. IEEE International Conference Robotics and Automation, 3888-3893.

F. Chaumette and S. Hutchinson. 2006. Visual servo control, part 1: Basic approaches. IEEE Robotics and Automation Magazine, 13(4):82-90.

F. Chaumette and S. Hutchinson. 2007. Visual servo control, part ii: Advanced approaches. IEEE Robotics and Automation Magazine, 14(1):109-118. 
L. Chen, W. Rong Sun, L. and, and X. Bian. 2004. Hybrid control of vision and force for MEMS assembly system. In IEEE International Conference on Robotics and Biometrics, 136-141, Shenyang, China.

M. B. Cohn, K. F. Böhringer, J. M. Noworolski, A. Singh, and C. G. Keller. 1998. Microassembly technologies for MEMS. In SPIE Microelectronic structures and MEMS for optical processing IV., 3513, 6-12, Santa Clara, CA.

A.I. Comport, E. Marchand, M. Pressigout, , and F. Chaumette. 2006. Real-time markerless tracking for augmented reality: the virtual visual servoing framework. IEEE Trans. on Visualization and Computer Graphics, 12(4):615-628.

C. del Corrala, P. J. Estebana, A. Albuta, Q. Zhoua, and H. N. Koivo. 2002. Influence of environment conditions on microassembly components and pick-and-place operations. Proceedings of the Fourth International Conference on Machine Automation, 267-274.

R. Devanathan, S. Wenting, S. Chai, and A. Shacklock. 2006. Multi view and multi scale image based visual servo for micromanipulation. Studies in Computational Intelligence, 8: $105-133$.

T. Drummond and R. Cipolla. 2002. Real-time visual tracking of complex structures. IEEE Trans. on Pattern Analysis and Machine Intelligence, 24(7):932-946.

S. Fatikow, A. Buerkle, and J. Seyfried. 1999. Automatic control system of a microrobotbased microassembly station using computer vision. In SPIE conference on Microrobotics and Microassembly, Vol. 3834, 11-22, Boston, Massachusetts, USA.

S. Fatikow, T. Wortmann, M. Mikczinski, C. Dahmen, and C. Stolle. 2009. Towards automated robot based nanohandling. In Proc. of IEEE Chinese Control and Decision Conference (CCDC), 5039-5044, China.

J. T. Feddema and R. W. Simon. 1998. Visual servoing and CAD-driven microassembly. IEEE Robotics and Automation Magazine, Vol. 5 (4):18-24.

M. Figl, C. Ede, J. Hummel, Felix Wanschitz, Rolf Ewers, Helmar Bergmann, and Wolfgang Birkfellner. 2005. A fully automated calibration method for an optical see-through headmounted operating microscope with variable zoom and focus. IEEE Transactions on Medical Imaging, Vol. 24, N 11:1492-1499.

M. A. Greminger and B. J. Nelson. 2004. Vision-based force measurement. IEEE Transactions on Pattern Analysis and Machine Intelligence, Vol. 26/N3:290-298.

M. A. Greminger and B. J. Nelson. 2005. A deformable object tracking algorithm robust to occlusions and spurious edges. In IEEE International Conference on Robotics and Automation, 1264-1269, Barcelana, Spain. 
R. Haralick, H. Joo, C. Lee, X. Zhuang, V Vaidya, and M. Kim. 1989. Pose estimation from corresponding point data. IEEE Trans on Systems, Man and Cybernetics, 19(6): $1426-1445$.

P.-J. Huber. 1981. Robust Statistics. Wiler, New York.

S. Hutchinson, G. Hager, and P. Corke. 1996. A tutorial on visual servo control. IEEE Trans. on Robotics and Automation, 12(5):651-670.

D. Jasper and S. Fatikow. 2009. High-speed position tracking for nanohandling inside scanning electron microscopes. In Proc. of IEEE Int. Conference on Robotics and Automation (ICRA), 508 - 513, Japan.

F. A. Kawaji and T. Fukuda. 2001. 3D attitude control system for biomicromanipulation. Proceeding of International Symposium of Micromechatronics and Human Science, 197202, Nagoya, Japan.

B. E. Kratochvil, L. X. Dong, and B. J. Nelson. 2009. Real-time rigid-body visual tracking in a scanning electron microscope. International Journal of Robotics Research, 28(4): $498-511$.

D. G. Lowe. 1991. Fitting parameterized three-dimensional models to images. IEEE Trans. on Pattern Analysis and Machine Intelligence, 13(5):441-450.

E. Malis, F. Chaumette, and S. Boudet. 1999. 2 1/2 D visual servoing. IEEE Trans. on Robotics and Automation, 15(2):238-250.

E. Marchand, F. Chaumette, F. Spindler, and M. Perrier. 2002. Controlling an uninstrumented manipulator by visual servoing. The International Journal of Robotics Research, IJRR, 21(7):635-648.

P. Martinet and J. Gallice. 1999. Position based visual servoing using a non-linear approach. In IEEE/RSJ Int. Conf. Intelligent Robots and Systems, 531-536, Kyongju, South Korea.

R. Murray, L. Zexiang, and S. Sastry. 1994. A Mathematical introduction to robotic manipulation. CRC Press.

B. J. Nelson, S. Ralis, Y. Zhou, and B. Vikramaditya. 1999. Force and vision feedback for robotic manipulation of the microworld. Experimental Robotics - VI-Lectures Notes in Control and Information Sciences, 250:433-442.

D. O. Popa and H. E. Stephanou. 2004. Micro and mesoscale robotic assembly. Journal of Manufacturing Process, 6 (1):52-71.

M. Probst, Ch. Hrzeler, R. Borer, and B. J. Nelson. 2009. A microassembly system for the flexible assembly of hybrid robotic MEMS devices. International Journal of Optomechatronics, 3(2):69-90. 
S. Ralis, B. Vikramaditya, and B. J. Nelson. 2000. Micropositioning of a weakly calibrated microassembly system using coarse-to-fine visual servoing strategies. IEEE Transactions on Electronics Packaging Manufacturing, Vol. 23 (2):123-131.

S. A. Stoeter, M. Probst, B. J. Nelson, and M. A. Iranzo. 2006. Improving tracking precision for microassembly. In Proceedings of the 2006 IEEE International Conference on Robotics and Automation, Orlando, Florida, USA.

W.T. Sun and T.C. Chin. 2004. Image-based visual servo for micromanipulation : a multiple-view and multiple-scale approach. In International Symposium on MicroNanomechatronics and Human Science, 341-346.

B. Tamadazte, S. Dembélé, and N. Le Fort-Piat. 2008a. A multiscale calibration of a photon video microscope for visual servo control: Application to micromanipulation. IEEE International Workshop on Robotic and Sensors Environments, 29-34, Ottawa, Canada.

B. Tamadazte, S. Dembélé, and N. Le Fort-Piat. 2009. A multiscale calibration of a photon videomicroscope for visual servo control: Application to MEMS micromanipulation and microassembly. Sensors and Transducers Journal, 5:37-52.

B. Tamadazte, S. Dembélé, G. Fortier, and N. Le Fort-Piat. 2008b. Automatic micromanipulation using multiscale visual servoing. In IEEE Conf. on Automation Science and Engineering, 977-982, Washington, USA.

X. Tao, H. Cho, and Y. Cho. 2005 Microassembly of peg and hole using active zooming. In SPIE, Society of Photo-Optical Instrumentation Engineers, Vol. 6052, 605204.1-605204.12, Bellingham, WA, USA.

R. Wechsung. 2002 Market analysis for microsystems 2002-2005. In A report from the NEXUS task force. MST.

W. Wilson, C. Hulls, and G. Bell. 1996. Relative end-effector control using cartesian positionbased visual servoing. IEEE Transaction on Robotics and Automation, 12:684-696.

G. Yang, J. Gaines, and B. J. Nelson. 2003. A surpervisory wafer-level 3D microassembly system for hybrid MEMS fabrication. Journal of Intelligent and Robotic Systems, 37: 43-68.

G. Yang, J. Gaines, and J. B. Nelson. 2005. Optomechatronic design of microassembly systems for manufacturing hybrid microsystems. IEEE Transactions on Industrial Electronics, 4: Vol. 52, 1013-1023.

K. Yesin and B. Nelson. 2005. A CAD-model based tracking system for visually guided microassembly. Robotica, 23:409-418. 
K. B. Yesin and B. J. Nelson. 2004. Robust CAD model-based visual tracking for 3D microassembly using image space potentials. In IEEE Int. Conf. on Robotics and Automation, 1868-1873, New Orleans, USA.

Q. Zhou and B. Chang. 2006. Microhandling using robotic manipulation and capillary self-alignment. In EEE/RSJ International Conference on Intelligent Robots and Systems, 5883-5888, Beijing, China.

Q. Zhou, C. Corral, P. Esteban, A. Albut, and H. Koivo. 2002. Environmental influences on microassembly. IEEE/RSJ International Conference on Intelligent Robots and System, $1760-1765$.

Y. Zhou and B. J. Nelson. 1999. Calibration of a parametric model of an optical microscope. Optical Engineering, 38(12):1989-1995. 


\section{Contents}

1 Introduction $\quad 2$

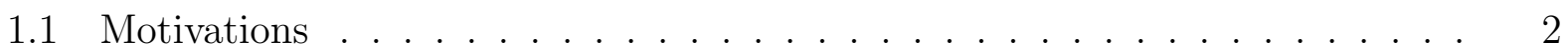

1.2 Previous research in MEMS assembly . . . . . . . . . . . . . . . . . . 3

1.3 Contributions and organization . . . . . . . . . . . . . . . . 4

2 Task specification and microsassembly workcell 5

2.1 Manipulated objects . . . . . . . . . . . . . . . . . . . 5

2.2 Microrobotic workstation . . . . . . . . . . . . . . . 6

2.2.1 Microhandling system . . . . . . . . . . . . . . . . 7

2.2 .2 Imaging system . . . . . . . . . . . . . . . . . . . . . . 9

2.3 Environment control system . . . . . . . . . . . . . . . . . . . 9

3 CAD model based tracking algorithm $\quad 10$

4 Assembly on the microscale by means of 3D visual control 12

4.1 Notations . . . . . . . . . . . . . . . . . . . . . . 12

4.2 Control law . . . . . . . . . . . . . . . . . . . . . . . 13

5 Experimental results $\quad 17$

5.1 Precision and robustness ...................... . . 17

5.2 Assembly cycle time . . . . . . . . . . . . . . . . . . . . . . . . . . . . . . . . . . .

5.3 Complex MEMS structures assembly . . . . . . . . . . . . . . 20

6 Conclusion and future perspectives 25

6.1 Conclusion . . . . . . . . . . . . . . . . . . . 25

6.2 Future perspectives . . . . . . . . . . . . . . . . . . . 28

7 Aknowledgements $\quad 29$

$\begin{array}{lr}\text { A Transformation definition } & 29\end{array}$

$\begin{array}{ll}\text { B Notations } & 30\end{array}$

$\begin{array}{ll}\text { C Index to multimedia extensions } & 31\end{array}$ 